\title{
INTERPOLACIÓN ESPACIAL DE LAS AMPLIFICACIONES DINÁMICAS DEL TERRENO EN EL VALLE DE MÉXICO
}

\author{
L E Pérez-Rocha ${ }^{(1)}$, M Ordaz Schroeder ${ }^{(2)}$ y E Reinoso Angulo ${ }^{(2)}$
}

\begin{abstract}
RESUMEN
Se describe un método para resolver un problema de interpolación espacial. Los datos son funciones de transferencia que describen las amplificaciones relativas del movimiento en la superficie del terreno del valle de México. Estas funciones se han obtenido a partir de los espectros de Fourier de acelerogramas producidos por varios temblores y registrados en decenas de sitios en las zonas de terreno firme, transición y lago. Se plantea un esquema estadístico para restringir la interpolación espacial de las funciones de transferencia mediante el uso de las técnicas de regresión bayesiana. Ello permite hacer una incorporación formal de la información que se tiene acerca de los efectos de sitio en la respuesta dinámica del terreno. Esta formulación suministra soluciones más estables y racionales que las que se obtendrían al seguir esquemas de regresión convencionales para la interpolación espacial de datos geofísicos. La utilidad práctica del enfoque propuesto es que permite cuantificar las amplificaciones dinámicas en sitios donde no hay instrumentos.
\end{abstract}

\begin{abstract}
A method to solve a problem of spatial interpolation is described. The data are transfer functions that describe the relative amplifications of the motion in the surface of the ground of the valley of Mexico. These functions have been obtained from the Fourier spectra of accelerograms produced by several earthquakes and registered in dozens of sites in the firm ground, transition and bed lake zones. A statistical scheme to restrict the spatial interpolation of transfer functions by means of the use of a bayessian regression technique is proposed. It allows to make a formal incorporation of the information that is had about the site effects in the dynamic response of the ground. This formulation provides more stable and rational solutions than those obtained when following conventional schemes of regression for the spatial interpolation of geophysical data. The practical utility of the proposed approach is that it allows to quantify the dynamic amplifications in sites where there are no instruments.
\end{abstract}

\section{INTRODUCCIÓN}

El interés por conocer la naturaleza del movimiento sísmico del terreno se ha promovido en la mayor parte de las zonas sísmicas altamente pobladas del mundo. Gracias al desarrollo tecnológico, se ha incrementado la capacidad de observación instrumental y, con ello, el conocimiento experimental en la

\footnotetext{
Artículo recibido el 14 de noviembre de 2008 y aprobado para su publicación el 27 de febrero de 2009. Se aceptarán comentarios y/o discusiones hasta cinco meses después de su publicación

${ }^{(1)}$ Investigador, Instituto de Investigaciones Eléctricas, Paseo de la Reforma 113, Temixco 62490, Morelos, México. E-mail: lepr@iie.org.mx

${ }^{(2)}$ Investigador, Instituto de Ingeniería, UNAM, Circuito Escolar, Ciudad Universitaria, 04510 México, D.F., México. E-mail: mors@pumas.iingen.unam.mx,$\underline{\text { ere@pumas.iingen.unam.mx }}$
} 
sismología y la ingeniería sísmica. En estas ciencias, buena parte del conocimiento es empírico, ya que se basa principalmente en la interpretación de los registros de movimiento fuerte. A su vez, esta interpretación está guiada por modelos teóricos e hipótesis sobre la mecánica de la ruptura y la propagación de ondas. Los esfuerzos por reducir el efecto destructivo de los grandes temblores han contribuido en el desarrollo de diversos esquemas para estimar el movimiento del terreno producido por posibles sismos futuros. En México se han desarrollado diversas técnicas, desde simples relaciones de atenuación hasta modelos semiempíricos para tomar en cuenta los efectos de fuente, de trayecto y de sitio.

A partir de una base de datos mundial que incluía registros de temblores mexicanos, Esteva y Villaverde (1973) obtuvieron relaciones llamadas leyes de atenuación, para aceleración y velocidad máximas del terreno. Bufaliza (1984) propuso expresiones similares obtenidas a partir de datos registrados exclusivamente en México. Posteriormente, Singh et al (1987) obtuvieron estas relaciones tomando sólo los datos registrados en la Ciudad Universitaria (CU) de la Ciudad de México. También se buscaron esquemas que suministren una mejor caracterización del movimiento, en particular, el contenido de frecuencias y la duración.

Por ejemplo, Castro et al (1988) construyeron un modelo para la regresión de las amplitudes del espectro de Fourier (EAF) en el sitio CU. Más adelante, Ordaz y Singh (1992) encontraron que existe un efecto de amplificación regional en el valle de México. Los autores señalan que aún en la zona firme del valle existen amplificaciones en un intervalo amplio de frecuencias que no se explican con los modelos más simples de fuente y trayecto, o que no tienen correlación con la atenuación sísmica observada en otras regiones. Tomando en cuenta estas evidencias, Ordaz et al (1994) construyeron un modelo de regresión de los EAF para el sitio CU. Esta formulación difiere de la propuesta por Castro et al (1988) porque en ella se incorpora toda la información existente haciendo uso del teorema de Bayes. De acuerdo con los autores, las regresiones son más estables y tienen una relación más estrecha con la naturaleza física del problema. Poco después, Pérez-Rocha et al (1996) encontraron que en la colección de acelerogramas registrados en CU pueden señalarse rasgos espectrales que probablemente provienen de la fuente sísmica y el trayecto. Sería de interés extender el modelo de Ordaz et al (1994) para tomar en cuenta estas peculiaridades de la fuente sísmica y/o del trayecto. Al parecer, los temblores que se generan en frente a las costas de Guerrero son más energéticos y particularmente destructivos para las estructuras de la Ciudad de México.

Un gran interés por reducir el peligro sísmico que enfrentan las estructuras y obras civiles de la Ciudad de México se despertó con los acontecimientos sísmicos de 1985. Como parte de las primeras iniciativas, se instalaron docenas de acelerómetros, principalmente en aquellos sitios en los que se han observado huellas de temblores intensos, tanto de destrucción de obras civiles como de rupturas en el terreno. Esta red instrumental, aquí llamada Red Acelerométrica de la Ciudad de México (RACM), cuenta en la actualidad con más de 100 acelerómetros sobre la superficie del terreno.

Con los primeros registros de la RACM, Singh et al (1988) obtuvieron una colección de funciones de transferencia empíricas (FTE) mediante los cocientes de las ordenadas de los EAF observados en sitios en las zonas de transición y del lago entre las del EAF del movimiento que se observó en cada caso en el sitio CU, localizado en terreno firme. Encontraron que en la zona del lago, el movimiento del terreno está fuertemente controlado por la respuesta dinámica de los estratos de suelo blando más superficiales. De acuerdo con los autores, típicamente se tienen espectaculares amplificaciones en aquellas frecuencias del movimiento que coinciden con las frecuencias dominantes del terreno y señalan que estas amplificaciones pueden estar presentes en los próximos grandes temblores. En efecto, Singh et al (1988) encontraron que el comportamiento dinámico de los depósitos lacustres es elástico y significativamente poco amortiguado, aún en sismos tan intensos como los de 1985. Esta hipótesis de elasticidad lineal permite hacer uso de las FTE obtenidas de los registros de sismos pequeños para predecir el movimiento producido por sismos intensos. El enfoque se debe a Ordaz et al (1989). En síntesis, se trata de una formulación completa para el 
cálculo de espectros de respuesta elásticos en sitios instrumentados mediante el uso de las FTE medidas en estos sitios y el EAF que se tendría en el sitio de referencia CU. Los autores compararon exitosamente las intensidades medidas en 1985 en estaciones de la zona de lago con las posdichas para estos sitios a partir de las FTE de Singh et al (1988) y el EAF observado en CU en 1985.

Con el análisis de los acelerogramas producidos por varios temblores de subducción, Reinoso (1991) identificó que el movimiento en el terreno firme tiene variaciones importantes. Señala que si las FTE se calculan con respecto al EAF promedio del terreno firme, la amplificación obtenida para la mayoría de los sitios es razonablemente constante de temblor a temblor, sin importar la magnitud, la distancia epicentral o el azimut de la incidencia. Para algunos sitios, esta amplificación puede ser explicada, razonablemente, mediante el modelo unidimensional de propagación de ondas de corte. Sin embargo, también hay evidencias de que los accidentes topográficos y geológicos pueden tener efectos importantes en las amplificaciones locales, en particular en los sitios cercanos a la frontera entre zonas de transición y de lago.

La estabilidad de las FTE medidas en sitios instrumentados estimuló el desarrollo de un modelo para cuantificar estas funciones de amplificación dinámica en sitios no instrumentados (Pérez-Rocha et al, 1991; Reinoso et al, 1992; Ordaz et al, 1992) mediante el uso de los modelos convencionales de interpolación espacial de datos geofísicos (Lancaster y Salkauskas, 1986; Pelto et al, 1988). Para los sectores densamente instrumentados se obtienen resultados excelentes. Sin embargo, se encontró que la inestabilidad numérica se incrementa dramáticamente en sitios alejados de la estaciones de la RACM y en las porciones del valle donde las condiciones geotécnicas locales presentan grandes variaciones espaciales. En este trabajo se recurre al teorema de Bayes para incorporar información adicional que permite superar las limitaciones debidas a la insuficiencia espacial de datos. A continuación se hace una descripción formal de este enfoque bayesiano para interpolar las FTE de sitios con coordenadas arbitrarias.

\section{INTERPOLACIÓN ESPACIAL DE DATOS GEOFÍSICOS}

Para la interpolación espacial de datos geofísicos se requiere un método para estimar los valores de una variable en un arreglo bidimensional irregular. Este problema ha sido resuelto por numerosos autores con diversos objetivos. En particular, Lancaster y Salkauskas (1986) señalan que en unos problemas se buscan tendencias que suavizan las variaciones espaciales de los datos, y que en otros, se hace el ajuste de formas funcionales para interpolar los datos. Los autores presentan resultados con los que ilustran diferencias espectaculares al resolver un problema arbitrario con los métodos más comunes.

Supóngase que en $n$ puntos $\left(\mathrm{x}_{i}, \mathrm{y}_{i}\right)$ se conocen los valores $z_{i}$ de un campo continuo en una región del dominio $\mathrm{x}-\mathrm{y}$. Si los datos son suficientes, se obtiene una buena descripción de las variaciones espaciales del campo mediante una representación en series de Taylor. En efecto, para determinar el valor $Z_{\mathrm{o}}$ del campo en el punto $\left(\mathrm{x}_{\mathrm{O}}, \mathrm{y}_{\mathrm{O}}\right)$, Pelto et al (1988) proponen el ajuste de los coeficientes de la siguiente forma funcional, deducida de un desarrollo de segundo orden,

$z(x, y)=\alpha_{0}+\alpha_{1}\left(x-x_{0}\right)+\alpha_{2}\left(y-y_{0}\right)+\alpha_{3}\left(x-x_{o}\right)^{2}+\alpha_{4}\left(y-y_{o}\right)^{2}+\alpha_{5}\left(x-x_{o}\right)\left(y-y_{o}\right)$

imponiendo que el error estándar sea mínimo. Para garantizar variaciones espaciales suaves, a cada dato $z_{i}$, se asigna un peso $w_{i}$ que decrece con la distancia entre las coordenadas $\left(\mathrm{x}_{i}, \mathrm{y}_{i}\right)$ y $\left(\mathrm{x}_{\mathrm{o}}, \mathrm{y}_{\mathrm{o}}\right)$. Si se busca que al interpolar en $\left(x_{i}, y_{i}\right)$ se tenga $z=z_{i}$, los autores recomiendan la forma 
$w_{i}=\frac{1}{\mathrm{r}_{i}^{2}}$

donde $\mathrm{r}_{i}^{2}=\left(\mathrm{x}_{i}-\mathrm{x}_{\mathrm{o}}\right)^{2}+\left(\mathrm{y}_{i}-\mathrm{y}_{\mathrm{o}}\right)^{2}$. Si el componente $i$-ésimo de la función de error es

$\varepsilon_{i}=\left(\alpha_{0}+\alpha_{1}\left(\mathrm{x}_{i}-\mathrm{x}_{\mathrm{o}}\right)+\alpha_{2}\left(\mathrm{y}_{i}-\mathrm{y}_{\mathrm{o}}\right)+\alpha_{3}\left(\mathrm{x}_{i}-\mathrm{x}_{\mathrm{o}}\right)^{2}+\alpha_{4}\left(\mathrm{y}_{i}-\mathrm{y}_{\mathrm{o}}\right)^{2}+\alpha_{5}\left(\mathrm{x}_{i}-\mathrm{x}_{\mathrm{o}}\right)\left(\mathrm{y}-\mathrm{y}_{\mathrm{o}}\right)-z_{i}\right) w_{i}$

se tiene el sistema de ecuaciones

$\mathbf{X}^{\mathrm{T}} \mathbf{W X} \boldsymbol{\alpha}=\mathbf{X}^{\mathrm{T}} \mathbf{W z}$

con la matriz $\mathbf{X}$ dada por

$$
\mathbf{X}=\left[\begin{array}{cccccc}
1 & \left(\mathrm{x}_{1}-\mathrm{x}_{\mathrm{o}}\right) & \left(\mathrm{y}_{1}-\mathrm{y}_{\mathrm{o}}\right) & \left(\mathrm{x}_{1}-\mathrm{x}_{\mathrm{o}}\right)^{2} & \left(\mathrm{y}_{1}-\mathrm{y}_{\mathrm{o}}\right)^{2} & \left(\mathrm{x}_{1}-\mathrm{x}_{\mathrm{o}}\right)\left(\mathrm{y}_{1}-\mathrm{y}_{\mathrm{o}}\right) \\
1 & \left(\mathrm{x}_{2}-\mathrm{x}_{\mathrm{o}}\right) & \left(\mathrm{y}_{2}-\mathrm{y}_{\mathrm{o}}\right) & \left(\mathrm{x}_{2}-\mathrm{x}_{\mathrm{o}}\right)^{2} & \left(\mathrm{y}_{2}-\mathrm{y}_{\mathrm{o}}\right)^{2} & \left(\mathrm{x}_{2}-\mathrm{x}_{\mathrm{o}}\right)\left(\mathrm{y}_{2}-\mathrm{y}_{\mathrm{o}}\right) \\
\vdots & \vdots & \vdots & \vdots & \vdots & \vdots \\
1 & \left(\mathrm{x}_{n}-\mathrm{x}_{\mathrm{o}}\right) & \left(\mathrm{y}_{n}-\mathrm{y}_{\mathrm{o}}\right) & \left(\mathrm{x}_{n}-\mathrm{x}_{\mathrm{o}}\right)^{2} & \left(\mathrm{y}_{n}-\mathrm{y}_{\mathrm{o}}\right)^{2} & \left(\mathrm{x}_{n}-\mathrm{x}_{\mathrm{o}}\right)\left(\mathrm{y}_{n}-\mathrm{y}_{\mathrm{o}}\right)
\end{array}\right]
$$

$\mathbf{W}$ es una matriz formada por los pesos $\mathrm{w}_{i j}$, con

$\mathrm{w}_{i j}=w_{i} w_{j} \delta_{i j} ; \quad \delta_{i j}=\left\{\begin{array}{ll}1 & i=j \\ 0 & i \neq j\end{array}, \quad i=j=1,2, \ldots n\right.$

$\boldsymbol{z}=\left(z_{1}, z_{2}, \cdots z_{n}\right)^{\mathrm{T}}$ es el vector de datos y $\boldsymbol{\alpha}=\left(\alpha_{0}, \alpha_{1}, \cdots \alpha_{5}\right)^{\mathrm{T}}$ es el vector de coeficientes, determinados mediante la expresión

$\boldsymbol{\alpha}=\left[\mathbf{X}^{\mathrm{T}} \mathbf{W X}\right]^{-1} \mathbf{X}^{\mathrm{T}} \mathbf{W z}$

Si $\quad v(x, y)=\left(1,\left(x-x_{o}\right),\left(x-x_{0}\right)^{2},\left(y-y_{o}\right),\left(y-y_{o}\right)^{2},\left(x-x_{o}\right)\left(y-y_{o}\right)\right)^{T}$, para las coordenadas $\left(\mathrm{x}_{\mathrm{o}}, \mathrm{y}_{\mathrm{o}}\right)$, se tiene

$z=v^{\mathrm{T}} \alpha=\alpha_{0}$

\section{MODELO BAYESIANO DE INTERPOLACIÓN ESPACIAL}

La técnica sugerida por Pelto et al (1988) se resume en hacer uso de la ec (4) para resolver un problema de interpolación. Esta ecuación es un esquema de regresión que se puede mejorar si se imponen restricciones adicionales a las que se tienen con el criterio del mínimo error. 
Supóngase que en el proceso de interpolación, el vector de errores para $\mathbf{n}$ sitios, $\boldsymbol{\varepsilon}=\left(\varepsilon_{1}, \varepsilon_{2}, \cdots \varepsilon_{\mathrm{n}}\right)^{\mathrm{T}}$, con el $i$-ésimo componente dado por la ecuación (3), tiene una distribución normal con media cero y matriz de covarianzas $\operatorname{COV}(\varepsilon)$. Los coeficientes $\boldsymbol{\alpha}=\left(\alpha_{0}, \alpha_{1}, \cdots \alpha_{5}\right)^{\mathrm{T}}$ también pueden interpretarse como variables aleatorias en el sentido de que nuestro conocimiento actual sobre ellos puede describirse haciendo uso de la teoría de probabilidades.

De acuerdo con el teorema de Bayes, la densidad de probabilidades que incluye el conocimiento previo de $\alpha$ y $\sigma$, así como la información contenida en los datos, cumple con la proporcionalidad

$$
p(\boldsymbol{\alpha}, \boldsymbol{\sigma} \mid \varepsilon) \propto p(\boldsymbol{\alpha}, \boldsymbol{\sigma}) L(\varepsilon \mid \boldsymbol{\alpha}, \boldsymbol{\sigma})
$$

donde $p(\alpha, \sigma)$ es la densidad previa conjunta con que se introduce el estado del conocimiento sobre los coeficientes $\alpha \mathbf{y} \sigma$ antes de examinar los datos, $p(\alpha, \sigma \mid \varepsilon)$ es la densidad conjunta posterior al examen y $L(\varepsilon \mid \alpha, \sigma)$ es la verosimilitud de los valores observados de $\varepsilon$ como una función de $\alpha$ y $\sigma$. En general, si la distribución de $\varepsilon \mid \alpha, \sigma$ es normal, y si los errores $\varepsilon$ tienen una matriz de covarianzas $\operatorname{COV}(\varepsilon)$, se tiene la siguiente forma analítica:

$$
\mathrm{L}(\varepsilon \mid \alpha, \sigma) \propto \mathrm{h}^{\mathrm{n} / 2} \exp \left(-\frac{\mathrm{h}}{2} \varepsilon^{\mathrm{T}} \boldsymbol{\Phi} \varepsilon\right)
$$

En esta expresión $h$ es la precisión (el inverso de la varianza $\sigma$ ) y $\Phi$ es una matriz adimensional, diagonal y conocida, que cumple con $\operatorname{COV}(\varepsilon)=h^{-1} \Phi^{-1}$. Nótese que la estructura asignada a $\operatorname{COV}(\varepsilon)$ implica que se ha supuesto que los errores en sitios diferentes son independientes entre sí, pero que no tienen la misma varianza.

Una aplicación de este teorema se presenta en Ordaz et al (1994). Los autores proponen un método de regresión para la atenuación de amplitudes de movimientos sísmicos a partir de una forma funcional deducida de un modelo teórico de fuente. En su formulación obtienen una expresión del error estándar similar a la ec (3). Señalan que el estado del conocimiento previo de los coeficientes $\alpha$ se puede describir mediante el uso de una función de densidad previa conjunta que sea conjugada natural del proceso. Esta función tiene la propiedad de tener la misma forma funcional que la densidad posterior conjunta. Extendiendo sus resultados (Broemling, 1985), puede demostrarse que el valor esperado de $\alpha$, posterior al examen de $\mathbf{z}$, es

$$
E(\boldsymbol{\alpha} \mid \boldsymbol{\varepsilon})=\mathbf{R}^{\prime \prime-1}\left(\mathbf{R}^{\prime} E(\boldsymbol{\alpha})+\mathbf{X}^{\mathrm{T}} \boldsymbol{\Phi} \mathbf{z}\right)
$$

donde la matriz $\mathbf{X}$ está dada en la ecuación 5 y el vector $E(\alpha)$ contiene los valores esperados previos de $\alpha$, en tanto que

$$
\mathbf{R}^{\prime}=\frac{\lambda^{\prime}}{r^{\prime}-1}(\operatorname{COV}(\alpha))^{-1}
$$

$\mathrm{y}$

$$
\mathbf{R}^{\prime \prime}=\mathbf{R}^{\prime}+\mathbf{X}^{\mathrm{T}} \boldsymbol{\Phi} \mathbf{X}
$$


donde $\operatorname{COV}(\alpha)$ es la matriz de covarianzas entre los coeficientes $\alpha$, antes de introducir la información contenida en los datos. Los parámetros $\lambda^{\prime}$ y $r^{\prime}$ están relacionados con la precisión $h$ a través de $E(h)=r^{\prime} / \lambda^{\prime}$ y $c^{2}(h)=1 / r^{\prime}$, donde $c$ denota coeficiente de variación. Estos parámetros reflejan la incertidumbre que se tiene a priori sobre $h$ y $\alpha$.

La función de densidad predictiva de $\mathbf{z}$, dado $\varepsilon$ y un nuevo vector de variables no aleatorias $\boldsymbol{v}$, es la distribución de $\mathbf{z}$, dados los parámetros $\alpha$ y $\sigma$, promediada con respecto a la distribución posterior $p(\alpha, \sigma \mid \varepsilon)$. Esta función de densidad es una $t$ de Student con $2 r^{\prime \prime}$ grados de libertad. Si para las coordenadas de interés $\left(\mathrm{x}_{\mathrm{O}}, \mathrm{y}_{\mathrm{O}}\right)$ se tiene que $\boldsymbol{v}=(1,0,0,0,0,0)^{\mathrm{T}}$, entonces

$$
E(z \mid \boldsymbol{\varepsilon}, \boldsymbol{v})=\boldsymbol{v}^{\mathrm{T}} E(\boldsymbol{\alpha} \mid \boldsymbol{\varepsilon})=E\left(\alpha_{0} \mid \boldsymbol{\varepsilon}\right)
$$

$\mathrm{y}$

$$
\operatorname{VAR}(z \mid \varepsilon, v)=\frac{\lambda^{\prime \prime}}{\left(r^{\prime \prime}-1\right) d}
$$

donde $\operatorname{VAR}(\cdot)$ denota varianza y

$$
\begin{aligned}
& r^{\prime \prime}=r^{\prime}+\frac{1}{2} \mathbf{J}^{\mathrm{T}} \boldsymbol{\Phi} \mathbf{J} ; \quad \text { con } \mathbf{J}^{\mathrm{T}}=(1,1,1,1,1,1) \\
& \lambda^{\prime \prime}=\lambda^{\prime}+\frac{1}{2}\left[E(\boldsymbol{\alpha})^{\mathrm{T}} \mathbf{R}^{\prime} E(\boldsymbol{\alpha})-E(\boldsymbol{\alpha} \mid \boldsymbol{\varepsilon})^{\mathrm{T}} \mathbf{R}^{\prime \prime} E(\boldsymbol{\alpha} \mid \boldsymbol{\varepsilon})+\mathbf{z}^{\mathrm{T}} \mathbf{\Phi} \mathbf{z}\right] \\
& d=1-\boldsymbol{v}^{\mathrm{T}}\left(\mathbf{R}^{\prime \prime}+\boldsymbol{v} \boldsymbol{v}^{\mathrm{T}}\right)^{-1} \boldsymbol{v}
\end{aligned}
$$

Las ecs (11) y (15) son el principal resultado de esta aplicación del teorema de Bayes. La ec (11) suministra una combinación formal del conocimiento previo con una solución similar a la que se obtiene de un ajuste convencional con el criterio del mínimo error. En efecto, si en la ec (11) se hace que los términos de $\operatorname{COV}(\alpha)=0$, lo cual significa que a priori no hay incertidumbre alguna sobre el valor de los coeficientes, se llega a que $E(\boldsymbol{\alpha} \mid \varepsilon)=E(\boldsymbol{\alpha})$. En cambio, cuando $\operatorname{COV}(\boldsymbol{\alpha})$ es infinita, se tiene que $\mathrm{E}(\boldsymbol{\alpha} \mid \boldsymbol{\varepsilon})=\left(\mathbf{X}^{\mathrm{T}} \boldsymbol{\Phi} \mathbf{X}\right)^{-1}\left(\mathbf{X}^{\mathrm{T}} \boldsymbol{\Phi} \mathbf{z}\right)$, que es el resultado usual de mínimos cuadrados. Por su parte, la ec (15) es una medida teórica de los errores que se cometen en la interpolación de $z$. En esta cantidad se toman en cuenta las incertidumbres asociadas con los errores aleatorios, con los datos y con los parámetros del modelo funcional.

Para aplicar las ecuaciones anteriores es necesario describir la información previa en términos de los parámetros $E(\alpha), \operatorname{COV}(\alpha), \lambda^{\prime} y r^{\prime}$. Además, hay que definir la matriz $\Phi$.

De los momentos previos de $\alpha$ sólo se tiene información sobre $\alpha_{0}$ que, de acuerdo con la ecuación 8 , es igual al valor que interesa predecir, es decir, $\mathbf{z}$. En vista de esto, se asignan valores muy grandes a los elementos de la diagonal de $\mathbf{C O V}(\alpha)$ en todos los renglones (que significa una gran incertidumbre sobre ellos) excepto en el primero, que corresponde a $\alpha_{0}$, sobre el que se tiene información previa que se 
describirá en las aplicaciones. Por otra parte, los valores de $E(\alpha)$ son irrelevantes, con excepción del primero, que corresponde a $\alpha_{0}$.

Para definir los valores de $\lambda$, $r^{\prime}$ y $\Phi$, se siguió el siguiente razonamiento. El hecho de que $\operatorname{COV}(\varepsilon)=h^{-1} \Phi^{-1}$ implica que se supone que en el sitio i, la varianza de $\varepsilon_{i}$ es $\left(h \Phi_{i i}\right)^{-1}$, es decir, que la varianza del error en los diferentes sitios tiene un factor de escala común a todos $(h)$ y otro factor, diferente para cada sitio, que define la precisión relativa entre observaciones en diferentes sitios. La distribución previa del factor de escala común queda definida al fijar las variables $\lambda^{\prime} y r^{\prime}$. No se dispone de información sobre esta variable, por lo que fijamos una distribución previa que introduce muy poca información $\left(r^{\prime}=2\right.$ y $\left.\lambda^{\prime}=1\right)$.

En vista de lo que se ha expuesto, la matriz $\Phi$ puede interpretarse como una matriz de pesos, equivalente a la matriz $\mathbf{W}$ de la ecuación 4. La manera de definir sus valores se describirá con detalle en los capítulos de aplicaciones. Sin embargo, en términos generales, se puede anticipar que cada elemento de la matriz $\Phi$ (recuérdese que se trata de una matriz diagonal) estará formado por dos componentes, el primero relacionado con la cercanía entre el sitio $i$ y el sitio para que se desea hacer interpolación, y otro relacionado con la precisión con que se conozca el valor de $\mathrm{z}$ en el sitio $i$. En este trabajo se han utilizado expresiones de la forma

$\Phi_{i i}^{-1}=z_{i}^{2}\left(c_{\mathrm{r}}^{2} \rho_{i}^{2}+c_{\mathrm{si}}^{2}\right)$

donde $c_{\mathrm{r}}$ es un coeficiente de variación espacial y $\rho_{\mathrm{i}}=\left(\mathrm{r}_{\mathrm{i}} / \mathrm{r}_{\mathrm{x}}\right)^{2}$ es un término adimensional con el que se toma en cuenta la distancia $\mathrm{r}_{\mathrm{i}}=\left(\left(\mathrm{x}_{\mathrm{i}}-\mathrm{x}_{\mathrm{o}}\right)^{2}+\left(\mathrm{y}_{\mathrm{i}}-\mathrm{y}_{\mathrm{o}}\right)^{2}\right)^{1 / 2}$ entre las coordenadas del dato $\mathrm{z}_{i} \mathrm{y}$ del sitio de interés, en tanto que $r_{x}$ es una distancia de referencia. Nótese que si $r_{i}$ crece, entonces $\Phi_{i i}$ crece, y por lo tanto, la influencia o peso del dato $z_{i}$ decrece. Por su parte, $c_{s i}$ es el coeficiente de variación de $\mathbf{z}$ en el sitio $i$, que mide con cuánta precisión se conoce el dato $z_{i}$.

Nótese que si $c_{\mathrm{r}}$ es suficientemente grande, el peso de cada dato sólo depende de las distancias $\mathrm{r}_{i}$. En cambio, si $c_{\mathrm{r}}=0$, la participación de cada dato se sujeta a su precisión relativa, dada por los coeficientes de variación $c_{s i}$.

\section{APLICACIONES A LOS DATOS SÍSMICOS REGISTRADOS EN EL VALLE DE MÉXICO}

La red actual de acelerómetros de la Ciudad de México cuenta con más de 100 estaciones en la superficie del terreno. En la figura 1 se indican las estaciones en operación y aquéllas que han sido retiradas pero que han suministrado datos para este estudio. Asimismo, se indican las zonas geotécnicas (terreno firme, zona de transición y zona de lago) y las principales vías de la ciudad. Los principales eventos sísmicos registrados con esta red de acelerómetros se indican en la tabla 1. La mayoría de ellos son de subducción, originados a centenas de kilómetros al sur de la ciudad.

El examen de los acelerogramas del primer sismo registrado por esta extensa red (ocurrido el 88/02/08) permitió a Singh et al (1988) reconocer los importantes efectos de amplificación dinámica debidos a las condiciones del terreno. Los autores proponen una descripción cuantitativa de estos efectos mediante funciones de transferencia empíricas (FTE). De acuerdo con Reinoso (1991), la estabilidad de las FTE, observadas de un temblor a otro, aumenta si se toma el cociente entre el espectro de amplitudes de Fourier (EAF) observado en cada sitio y el EAF promedio observado en las estaciones de terreno firme. 
Posteriormente, el análisis de los registros producidos por los sismos de fallamiento normal ocurridos los días 94/05/23 y 94/12/10, permitió identificar que las diferencias en las FTE producidas por temblores de distinto origen, son comparables a las que se tienen entre las FTE producidas por temblores del mismo origen (Pérez-Rocha et al, 1995). Al parecer, esto puede deberse a que la distancia epicentral de estos eventos es comparable con la distancia típica de los sismos de subducción, tomando como referencia a la Ciudad Universitaria. Para la mayoría de los sitios, el análisis estadístico de las FTE sugiere que gran parte de los efectos de amplificación estará presente en eventos futuros.

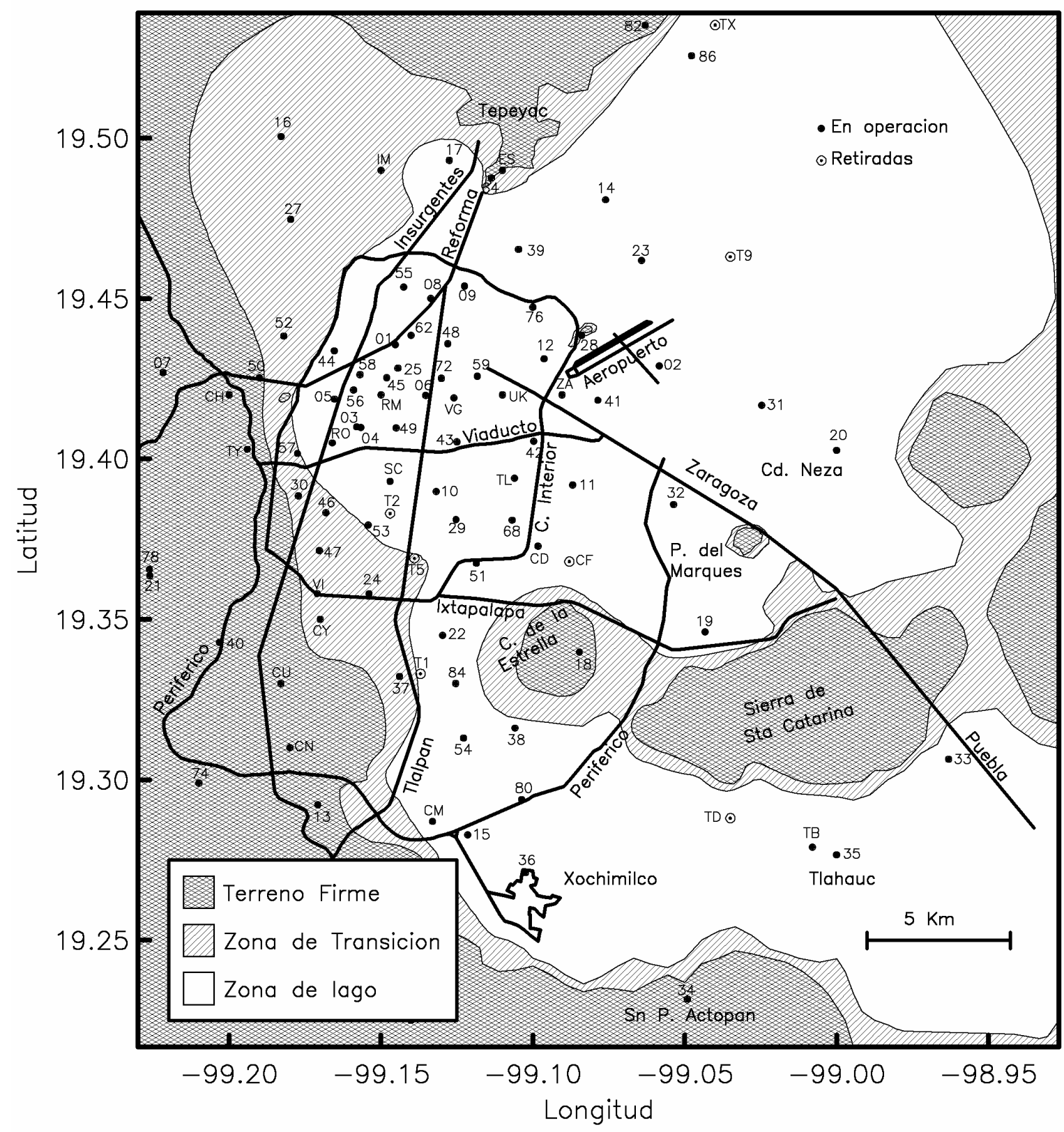

Figura 1 Red Acelerométrica de la Ciudad de México (RACM). Se indican las tres zonas geotécnicas (terreno firme, zona de transición y zona de lago) y las principales vías de la ciudad. 
En este trabajo se presenta una formulación para cuantificar las amplificaciones dinámicas del terreno en sitios no instrumentados. El método se basa en hacer una interpolación espacial de las ordenadas espectrales de las FTE descritas en términos de periodos adimensionales (Pérez-Rocha et al, 1991; Reinoso et al, 1992; Ordaz et al, 1992). Esta representación, que es exacta para el modelo unidimensional de propagación de ondas de corte, consiste en normalizar los periodos de la excitación con respecto al periodo dominante del terreno Ts. Por definición, los máximos de estas funciones se presentan en el periodo adimensional unitario. Para un sitio arbitrario se inicia con la interpolación del periodo dominante del terreno Ts. Para cada abscisa adimensional se calcula la ordenada correspondiente de una función de transferencia a priori FTP que se introduce en el esquema bayesiano para la interpolación de la función de transferencia a posteriori. La FTE objetivo se obtiene al escalar las abscisas de la función $a$ posteriori con Ts.

Tabla 1 Sismos estudiados para calcular periodos dominantes del terreno y funciones de transferencia empíricas (FTE).

\begin{tabular}{cccccc}
\hline \multirow{2}{*}{ FECHA } & \multirow{2}{*}{ ORIGEN } & $\begin{array}{c}\text { MAGNITUD } \\
\text { M LATITUD }\end{array}$ & $\begin{array}{c}\text { LONGITUD } \\
\text { DISTANCIA }\end{array}$ & $\begin{array}{c}\text { N } \\
\text { Mm }\end{array}$ \\
\hline $85 / 09 / 19$ & Subducción & 8.1 & 18.14 & 102.71 & 295 \\
$88 / 02 / 08$ & Subducción & 5.8 & 17.00 & 101.00 & 289 \\
$89 / 04 / 25$ & Subducción & 6.9 & 16.00 & 99.00 & 304 \\
$89 / 05 / 02$ & Subducción & 5.0 & 16.30 & 99.35 & 305 \\
$90 / 05 / 11$ & Subducción & 5.3 & 17.15 & 100.85 & 295 \\
$90 / 05 / 31$ & Subducción & 6.1 & 17.15 & 100.85 & 295 \\
$93 / 10 / 24$ & Subducción & 6.7 & 16.50 & 99.00 & 310 \\
$94 / 05 / 23$ & Normal & 6.0 & 18.03 & 100.57 & 205 \\
$94 / 12 / 10$ & Normal & 6.3 & 18.02 & 101.56 & 290 \\
$95 / 0914$ & Subducción & 7.3 & 16.80 & 98.60 & 300 \\
$95 / 10 / 09$ & Subducción & 8.0 & 18.60 & 104.00 & 560 \\
\hline
\end{tabular}

En este esquema probabilista de interpolación, se busca que al fijar un coeficiente de variación $c$, se de mayor influencia a los periodos dominantes más largos y a las ordenadas espectrales de mayor amplitud, especialmente en zonas insuficientemente instrumentadas. Por ello, se hace que la variable $z$ sea el inverso de los datos que interesa interpolar, es decir, el inverso de los periodos dominantes del terreno y de las ordenadas de las FTE. De esta forma, las varianzas $\sigma^{2}=c^{2} z^{2}$ menores (mayor peso o influencia) corresponden a los valores menores de $z$. En adelante, $z$ es el inverso de los datos, aunque se le refiere como el dato mismo y no como su inverso. Asimismo, el coeficiente de variación se refiere a la estadística de los valores inversos.

\section{PERIODOS DOMINANTES DEL TERRENO}

Haciendo uso de todos los registros de cada estación de la RACM, se calcularon los promedios de los periodos dominantes del terreno obtenidos de los componentes horizontales NS y EW. Estos valores se determinaron mediante la localización de los máximos espectrales de las FTE medidas en estas direcciones. Ya que se cuenta con $m_{i}$ observaciones independientes del periodo dominante en cada sito con coordenada $\left(\mathrm{x}_{i}, \mathrm{y}_{i}\right)$, se tiene una varianza estadística $\sigma_{m_{i}}^{2}$ de esta cantidad (en realidad, de su inverso $z_{i}$ ). En general, se cuenta con diferente número de observaciones en cada sitio. Ello se puede tomar en 
cuenta utilizando la teoría bayesiana para la estimación de los parámetros de una distribución normal, especialmente en lo que se refiere a la varianza. Las ecuaciones correspondientes son las siguientes (Broemling, 1985):

$\operatorname{VAR}(z \mid \varepsilon)=\frac{\lambda^{\prime \prime}}{\left(r^{\prime \prime}-1\right) d}$

$r^{\prime \prime}=r^{\prime}+\frac{m_{i}}{2}$

$\lambda^{\prime \prime}=\lambda^{\prime}+\frac{m_{i}}{2} \sigma_{m i}^{2}$

$d=\frac{m_{i}}{m_{i}+1}$

En vista de lo anterior,

$\sigma_{\mathrm{si}}^{2}=\left(\left(\mathrm{c}_{\mathrm{mo}} \mathrm{z}_{\mathrm{i}}\right)^{2}+\frac{\mathrm{m}_{\mathrm{i}}}{2} \sigma_{\mathrm{mi}}^{2}\right)\left(\frac{\mathrm{m}_{\mathrm{i}}+1}{\mathrm{~m}_{\mathrm{i}}}\right)\left(\frac{2}{2+\mathrm{m}_{\mathrm{i}}}\right)$

Para cada estación en las zonas de transición y de lago se calcularon los promedios de los periodos dominantes en las direcciones NS y EW ( $\mathrm{Ts}_{\mathrm{NS}} \mathrm{y} \mathrm{Ts}_{\mathrm{EW}}$, respectivamente) y el promedio entre ellos (Ts). También se calcularon los coeficientes de variación que se tienen entre los periodos $\operatorname{Ts}_{\mathrm{NS}} \mathrm{y} \mathrm{Ts}_{\mathrm{EW}}$ con respecto a la media Ts (denotado con $C_{\mathrm{H}_{i}}$ ), así como los coeficientes de variación después de $m_{i}$ observaciones $\left(c_{m_{i}}\right)$ y los que se obtienen con la ec (24) con el propósito de compensar el hecho de que el número de observaciones varía entre estaciones $\left(c_{\mathrm{Si}}\right)$. Los resultados más significativos se presentan en la figura 2 mediante histogramas de los coeficientes de variación al medir los periodos en las direcciones NS y EW $\left(c_{\mathrm{H}}\right)$, los obtenidos de temblor a temblor $\left(c_{m}\right)$ y los modificados $\left(c_{\mathrm{S}}\right)$, al introducir el coeficiente de referencia $c_{m_{0}}$. Se puede verificar que los periodos dominantes del terreno en una dirección tienen variaciones mayores de temblor a temblor, que las que se tienen entre los periodos medidos en las dos direcciones horizontales. En efecto, las diferencias entre los periodos dominantes del terreno medidos en dirección NS y EW, se traducen en coeficientes de variación $C_{\mathrm{H}}<0.01$ para más del $40 \%$ de las estaciones. Para casi el $100 \%$ se tienen coeficientes $c_{\mathrm{H}}<0.05$. Estos coeficientes son sensiblemente menores que los coeficientes $C_{m}$ (al centro de la figura 2), los cuales miden diferencias de temblor a temblor y alcanzan valores cercanos a 0.1 y tienen media $c_{m}=0.05$. Estos coeficientes describen las variaciones de los periodos dominantes de temblor a temblor. Para calcular los coeficientes $C_{\mathrm{S}}$, ilustrados en la parte derecha de la figura 2, el coeficiente de referencia $c_{m_{\mathrm{O}}}$ se hizo igual al valor de la cota superior de $c_{m}$, es decir, $c_{m_{\mathrm{O}}}=0.1$. Con esta corrección, los nuevos coeficientes $c_{\mathrm{S}}$ varían entre $0.05 \mathrm{y} 0.12$, en tanto que el valor promedio es $c_{\mathrm{S}}=0.08$. Con base en estos resultados, para las estaciones de terreno firme se fijó $\mathrm{Ts}=0.5 \mathrm{~s}$ con $c_{\mathrm{S}}=0.005$. Este valor de $c_{\mathrm{S}}$ es la décima parte del valor promedio para las zonas de transición y de lago (cercano a $c_{m}=0.05$ ), ya que se esperan variaciones no significativas de los periodos dominantes en la zona geotécnica firme. Finalmente, como los coeficientes que miden las diferencias de temblor a temblor $\left(c_{m}\right)$ son sistemáticamente mayores que los que miden las diferencias entre los dos 
componentes horizontales ortogonales $\left(c_{\mathrm{H}}\right)$ se decidió trabajar sólo con el periodo dominante promedio de los dos componentes horizontales.
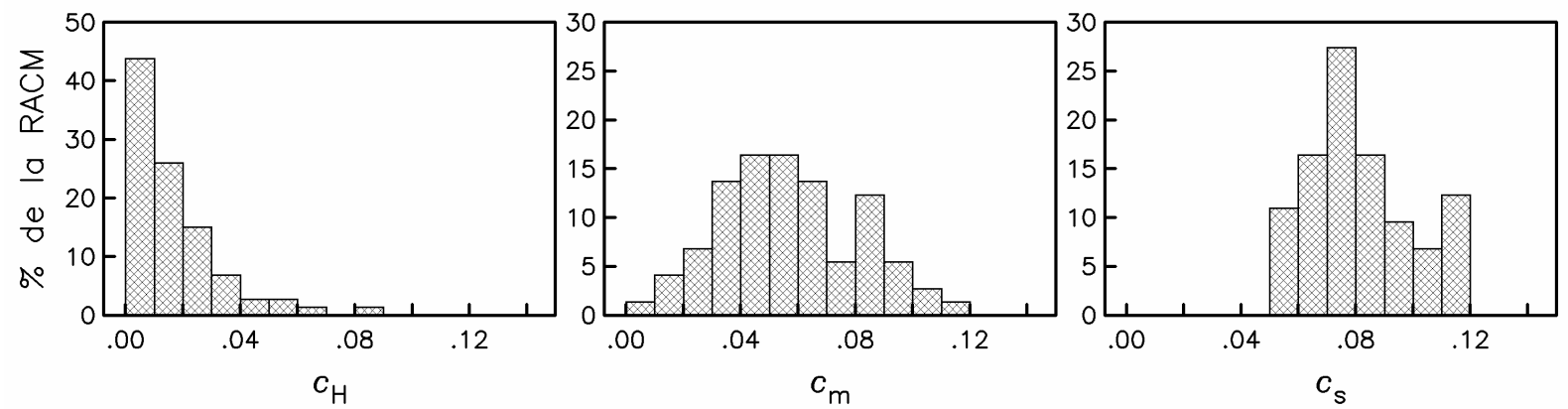

Figura 2 Histogramas de los coeficientes de variación de los periodos dominantes del terreno $c_{\mathrm{H}}$ (diferencias entre las direcciones NS y EW), $c_{m}$ (diferencias de temblor a temblor) y $c_{\mathrm{S}}$ (con $c_{m_{\mathrm{O}}}=0.1$ según la ec 24)

Estos resultados son medidas estadísticas del periodo dominante del terreno que se obtuvieron del análisis de los acelerogramas de varios temblores registrados en más de 70 sitios en las zonas de transición y de lago. También se cuenta con una colección de periodos dominantes obtenidos por Lermo et al (1988) a partir del registro de microtremores en cientos de sitios. Se admitió que los periodos obtenidos de los registros de microtremores son más inciertos que los que se obtienen de los registros de movimiento fuerte. Se les asignó un coeficiente de variación $c_{m}=0.15$, que es ligeramente mayor que el valor máximo reportado para las estaciones de la RACM $\left(c_{m}=0.12\right)$. Finalmente, para confinar la zona del terreno firme se colocaron puntos de control a lo largo de la frontera con la zona de transición, espaciados a cada $3 \mathrm{~km}$, aproximadamente. Al igual que en los sitios instrumentados del terreno firme, en estos sitios hipotéticos se ha fijado Ts $=0.5 \mathrm{~s}$ y $c_{\mathrm{S}}=0.005$. En ausencia de información previa, la ec (11) adquiere la forma

$$
\mathrm{E}(\boldsymbol{\alpha} \mid \boldsymbol{\varepsilon})=\left(\mathbf{X}^{\mathrm{T}} \boldsymbol{\Phi} \mathbf{X}\right)^{-1}\left(\mathbf{X}^{\mathrm{T}} \boldsymbol{\Phi} \mathbf{z}\right)
$$

El coeficiente de variación $c_{\mathrm{r}}$ se calibró de forma que el promedio de los coeficientes de variación esperados después de interpolar los periodos dominantes en los sitios instrumentados $\mathrm{c}_{\mathrm{z}}=\mathrm{z}^{-1}(\operatorname{VAR}(\mathrm{z} \mid \boldsymbol{\varepsilon}, \boldsymbol{v}))^{1 / 2}$, fuera comparable con el promedio de los coeficientes de variación $c_{\mathrm{S}}$. El parámetro $r_{x}$ relacionado con la ec (19) se hizo unitario. En la figura 3 se muestran cuatro coeficientes de variación $\left(c_{a}, c_{b}, c_{c}, c_{d}\right)$ que dependen de $c_{\mathrm{r}}$. Estos coeficientes son el promedio de los coeficientes calculados para las estaciones de la RACM en zonas de transición y de lago. El coeficiente $c_{a}$ se refiere a la diferencia medida entre los datos y los valores interpolados, en tanto que el coeficiente $c_{b}$ es el valor esperado, obtenido al hacer uso de la ec (15). Con el propósito de conocer los errores que se tendrían en sitios no instrumentados se calcularon los coeficientes medido $c_{C}$ y predicho $c_{d}$. En esta modalidad, llamada en adelante sin dato, se extrajo el dato de cada sitio al momento de la interpolación.

De acuerdo con estos resultados, el coeficiente óptimo está cerca de $C_{\mathrm{r}}=0.3$. Para este valor, los coeficientes de variación sin dato $\left(c_{C}\right.$ y $\left.c_{d}\right)$ son muy parecidos, cercanos a 0.15 , en tanto que los coeficientes de variación con dato $\left(c_{a}\right.$ y $\left.c_{b}\right)$ ya se han reducido a valores menores que la media estadística $c_{\mathrm{S}}=0.08$ (figura 2). En la figura 4 se confirman estas tendencias. Se trata de los histogramas de los 
coeficientes $c_{a}, c_{b}, c_{c}$ y $c_{d}$ con $c_{\mathrm{r}}=0.3$. Los errores con dato, medidos $\left(c_{a}\right)$ y esperados $\left(c_{b}\right)$, en promedio, son cercanos a $2 \%$ y $6 \%$, respectivamente, mientras que los errores sin dato son, en promedio, del $15 \%$.

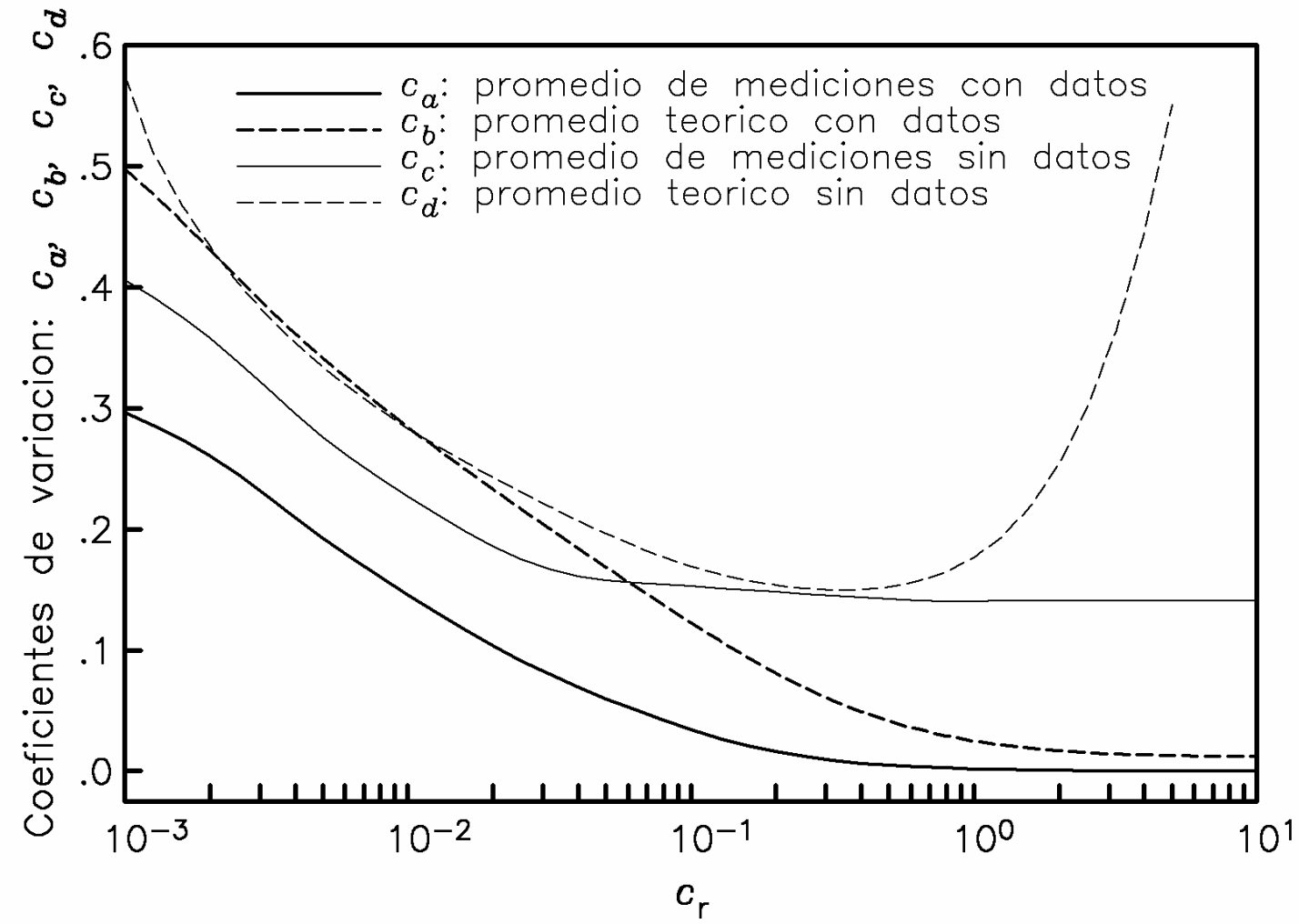

Figura 3 Coeficientes de variación relativos al error en la interpolación de $z$ v.s. $c_{r}$
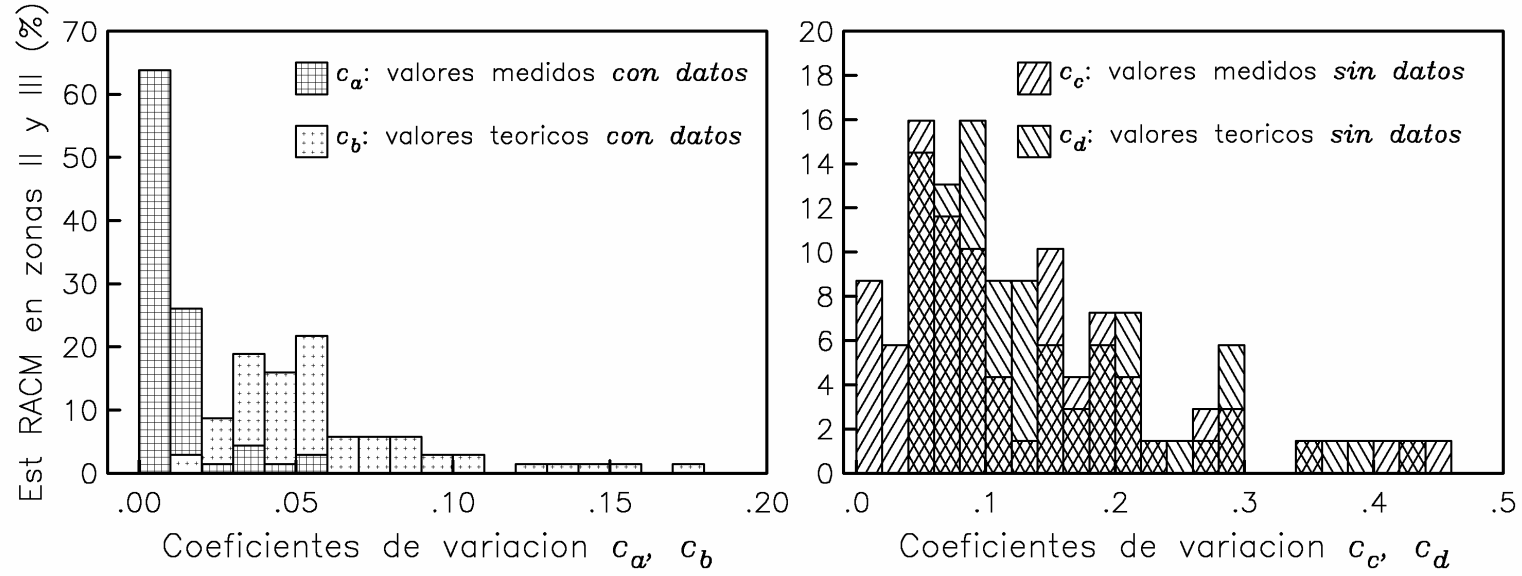

Figura 4 Histogramas de los coeficientes $c_{a}, c_{b}, c_{c}$ y $c_{d}$ 
Finalmente, se hizo un examen del sesgo que se introduce con este modelo de interpolación. En esta etapa interesa conocer qué desviación se tiene en la interpolación de la variable $Z$ (que es el inverso del periodo Ts), considerando la totalidad de los datos $(N)$. Para ello, se define como sesgo $c_{X}$ al promedio de las diferencias que se tienen en la interpolación de cada dato $Z_{i}$, en función de las coordenadas del sitio $i$ y del coeficiente $C_{r}$, es decir

$c_{x}\left(c_{r}\right)=\frac{1}{N} \sum_{i=1}^{N} \frac{\left(z\left(\mathrm{x}_{i}, \mathrm{y}_{i}, c_{r}\right)-z_{i}\right)}{z_{i}}$

Para este coeficiente también se calcularon las modalidades con dato $c_{S a}$ y $\sin$ dato $C_{S C}$. La variación de estos coeficientes con respecto a $c_{\mathrm{r}}$ se ilustra en la figura 5. Prácticamente, estas curvas son asintóticas a 0.0 y 0.12 , respectivamente, para valores grandes de $c_{\mathrm{r}}$. Aquí, el sesgo positivo indica que los periodos dominantes interpolados, sistemáticamente, son menores que los periodos dominantes reales.

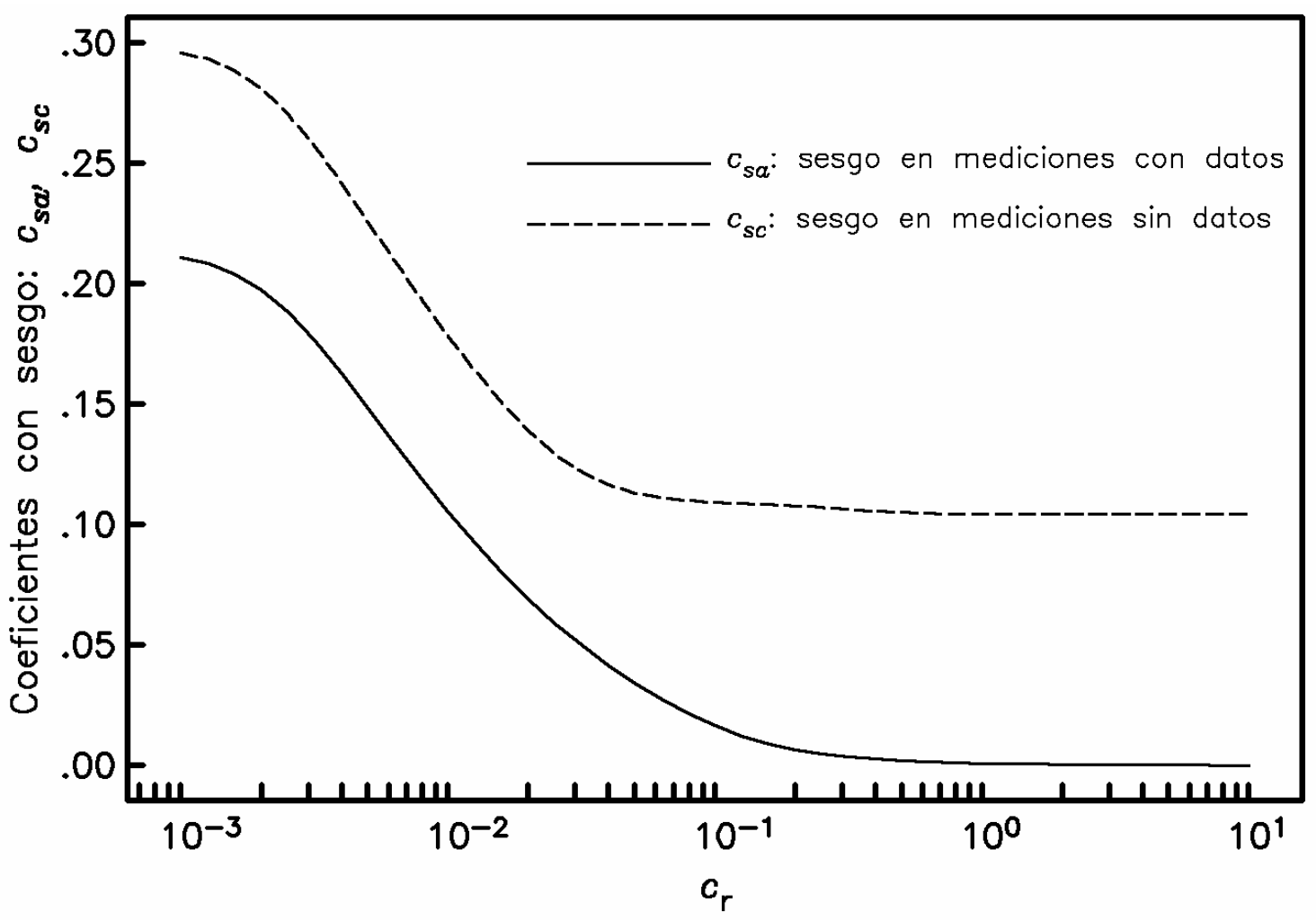

Figura 5 Coeficientes de variación sesgados v.s. $c_{r}$

En la figura 6 se muestra un mapa con curvas de periodos dominantes del terreno. Estos contornos se obtuvieron al evaluar el periodo dominante en los nodos de un arreglo cartesiano, equiespaciados a 0.5 $\mathrm{km}$ en las direcciones NS y EW. Los errores esperados se indican en el mapa de la figura 7. También se indican las estaciones de la RACM y los sitios donde se midieron los microtremores incluidos en este estudio. Dos de las zonas de mayor incertidumbre se centran en los cerros Peñón de los Baños y Peñón del 
Marqués. Ello se debe a la insuficiencia de instrumentos para describir grandes variaciones del periodo dominante en distancias del orden de centenas de metros. Las grandes zonas de alta incertidumbre situadas al NE del Lago de Texcoco y SE del Lago Xochimilco-Chalco obedecen al mismos efecto en una escala de kilómetros. Los errores máximos son del 70\%.

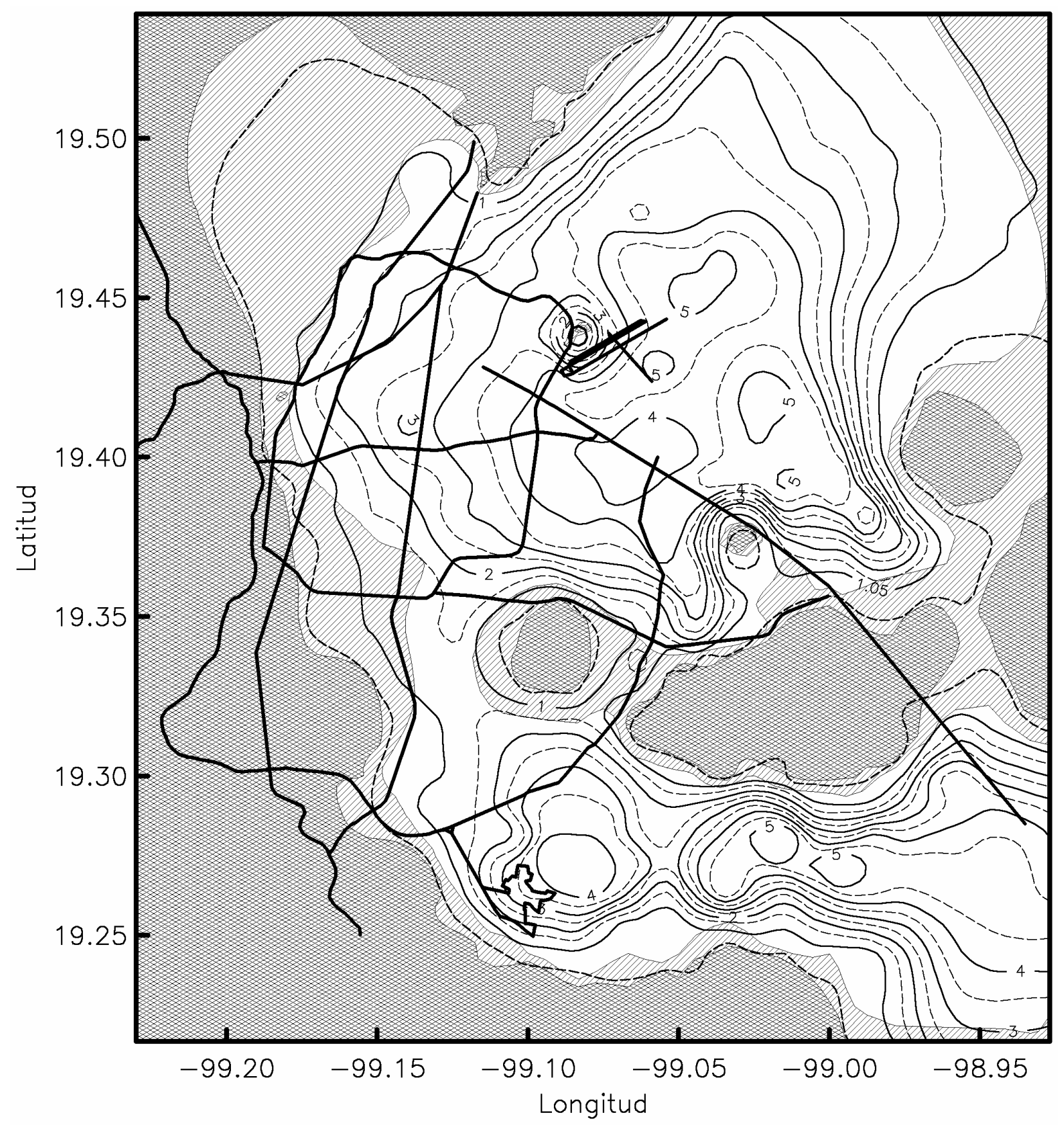

Figura 6 Mapa de isoperiodos dominantes del terreno. Se indican curvas desde $\mathrm{Ts}=0.5 \mathrm{~s}$ hasta Ts $=5.0 \mathrm{~s}$, con incrementos de $0.5 \mathrm{~s}$ 


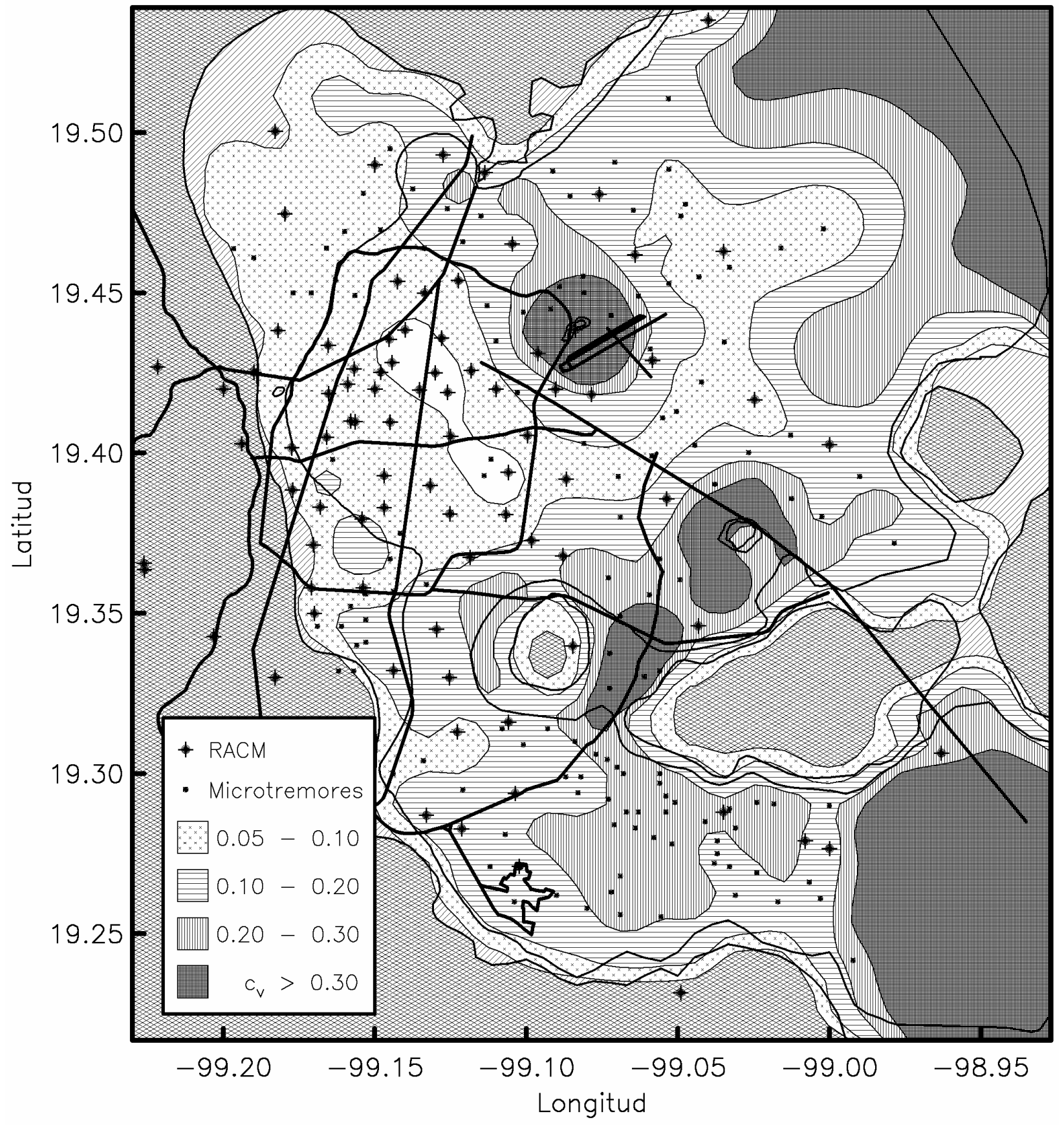

Figura 7 Coeficientes de variación esperados en la interpolación de periodos dominantes del terreno 


\section{FUNCIONES DE TRANSFERENCIA EMPÍRICAS}

La mayor parte del conocimiento sobre la respuesta sísmica de los depósitos lacustres del valle de México se ha adquirido después de los episodios sísmicos vividos en 1985. El análisis de las FTE obtenidas de los registros sísmicos permite ilustrar que los espectaculares efectos de sitio observados en la zona del lago no se explican completamente con la teoría de propagación unidimensional de ondas elásticas. Tampoco se explican con modelos numéricos de mayor complejidad, aunque estos reflejan la naturaleza tridimensional del movimiento y los sorprendentes efectos que pueden tener en él las irregularidades topográficas y geológicas de pequeña y gran escala (Sánchez-Sesma et al, 1992). Este es el conocimiento que se tiene sobre las amplificaciones dinámicas de los depósitos lacustres, previo a la interpolación espacial de los datos. De acuerdo con la formulación, para cada sitio de interés se introduce una descripción cuantitativa de este conocimiento como función de transferencia a priori (FTP). La FTP no debe ser significativa en la solución cuando se trata de interpolar FTE en zonas densamente instrumentadas. Ante la insuficiencia espacial de datos, se busca reducir las inestabilidades numéricas que obstaculizan las soluciones razonables y físicamente plausibles. También es de interés que la solución preserve las peculiaridades observadas sólo en el valle de México. De hecho, mientras menor sea la diferencia entre la FTP y las FTE datos, menores serán los errores debidos a la interpolación.

\section{Funciones de Transferencia a priori (FTP)}

Las funciones que se tomarán como funciones a priori tienen la misma estructura que las FTE. Cada una de ellas es función de la frecuencia, o bien, periodo de la excitación. Además, se buscan funciones de la frecuencia que, particularmente, también dependen del periodo dominante del terreno. Considérese, por ejemplo, la solución del modelo unidimensional de propagación de ondas en un sólo estrato de periodo Ts. Puede demostrarse que la función de transferencia teórica que proviene de un modelo de este tipo es

$\mathrm{H}(\omega, \mathrm{Ts})=\left|\frac{1}{\cos \left(\frac{\omega \mathrm{Ts}}{4}\right)+\mathrm{i} \eta \operatorname{sen}\left(\frac{\omega \mathrm{Ts}}{4}\right)}\right|$

El primer paso fue formar una base de datos con las FTE promedio de varios temblores en cada sitio instrumentado, escritas con abscisas normalizadas con respecto al periodo dominante del terreno Ts, que se llamará $\tau$. De esta forma los máximos de las FTE se encuentran en la abscisa igual a uno. El número de estas abscisas normalizadas es igual a 201, logarítmicamente equiespaciadas entre $10^{-1.5}$ y $10^{1.5}$. Las ordenadas de la base de datos se denotarán con $A_{i j}$, donde el primer índice indica periodo de excitación normalizado (por lo que $i$ está entre 1 y 201) y el segundo índice corre para las diferentes estaciones de registro (por lo que $j$ está entre 1 y N).

El segundo paso fue construir un modelo de interpolación para las ordenadas de las FTP como función del periodo de la excitación, T, y el periodo de sitio Ts, lo cual corresponde a $\tau_{k}=\mathrm{T}_{k} / \mathrm{Ts}$. Se hace notar que esta interpolación puede hacerse sólo para los 201 valores fijos de $\tau$, por lo que el valor de $\mathrm{T}_{k}$ no puede escogerse de manera arbitraria.

Una vez fijo $\tau_{k}$ se seleccionaron, de la base de datos, todas las ordenadas que corresponden a la $k$ ésima abscisa, asociada cada una a un diferente valor de Ts. A estas ordenadas se les denotará como 
$z_{j}=1 / A_{k j}$. A continuación se formula un modelo de interpolación similar al descrito en la ecuación 1 , pero sólo para una dimensión, que adopta la siguiente forma:

$z\left(x_{j}\right)=\beta_{0}+\beta_{1} x_{j}+\beta_{2} x_{j}^{2}$

donde $x_{j}=\log \left(\mathrm{Ts}_{j} / \mathrm{Ts}_{\mathrm{o}}\right), \mathrm{Ts}_{j}$ es el periodo predominante en el sitio $j \mathrm{y} \mathrm{Ts_{o }}$ es el periodo predominante del sitio cuya FTP se quiere determinar. Los coeficientes $\beta_{0}, \beta_{1}$ y $\beta_{2}$ son desconocidos. En vista de que se carece de información previa, la solución a esta interpolación está dada en la ecuación 7 , con una matriz de pesos $\mathbf{W}$ que, como se describe en la ecuación 6, es diagonal, con elementos denotados con $\sigma_{j}^{2}$, que adopta la siguiente forma:

$\sigma_{j}^{2}=z_{j}^{2}\left(c_{\mathrm{T}}^{2} \chi_{j}^{2}+c_{m_{j}}^{2}\right)$

donde $\chi_{\mathrm{j}}=\left(\log \left(\mathrm{Ts}_{j} / \mathrm{Ts}_{\mathrm{o}}\right)\right)^{2}$ mide la cercanía entre los periodos del sitio $j$ y del sitio de interés, $c_{m j}$ es el coeficiente de variación estadístico de temblor a temblor en el sitio $j$ y $c_{\mathrm{T}}$ es un coeficiente que mide la importancia relativa con que participa la cercanía entre periodos en el esquema de interpolación. Los valores de $C_{m j}$ oscilan, para las diferentes estaciones, entre 0.15 y 0.3 con media igual a 0.2 ; para las estaciones que sólo han registrado un evento sísmico se hizo $C_{m j}=0.3$.

El valor de $c_{\mathrm{T}}$ se ajustó de forma que se obtengan FTP con variaciones suaves en el dominio de Ts y que los errores relativos que se obtienen al comparar las FTE promedio de varios temblores con las FTP (denotados como $C_{p j}$ ) fueran comparables, para la mayoría de las estaciones, con los valores de $c_{m j}$, es decir, los coeficientes de variación observados. Estas FTP se calcularon para los periodos Ts de las estaciones que produjeron FTE y poder calcular errores entre estas FTE y FTP. Se obtuvieron coeficientes $c_{p j}$ que varían entre 0.1 y 0.5 , con media 0.23 . En la figura 8 se ilustran los histogramas de los coeficientes $C_{p j}$ (diferencias entre FTE promedio de varios temblores y FTP) y $c_{m j}$ (diferencias entre las FTE de varios temblores). Nótese que existe un incremento en los coeficientes $C_{p j}$ que obedece a que las FTE de algunas estaciones tienen grandes diferencias con el resto (en escalas normalizadas $\tau=\mathrm{T} / \mathrm{Ts}$ ). El coeficiente $c_{p j}$ es un indicador de la precisión de la FTE de un sitio debida a dos conceptos. Uno es el introducir las variaciones que se tienen de un temblor a otro. El otro puede interpretarse como la comparación de estas FTE con las FTE de otros sitios con periodo dominante similar.

Se optó por llevar a cabo esta interpolación en ambos componentes horizontales por separado, porque a diferencia de los periodos dominantes del terreno, se observó que las variaciones que se tienen entre las FTE de ambos componentes son comparables o mayores que las que se tienen para un componente dado de un temblor a otro. Esto se puede apreciar en la figura 9, donde se comparan las FTE de varios temblores y las FTE promedio, para los sitios SCT (Secretaria de Comunicaciones y Transportes) y CAO (Central de Abastos Oficinas). En la figura 10 se ilustran los dos componentes de las FTP que se tiene para sitios con periodos dominantes $\mathrm{Ts}=0.5,0.6, \ldots, 5.4$ (s). Además de que se tienen diferencias significativas entre ambos componentes, estas FTP guardan diferencias aún mayores con la solución que predice el modelo unidimensional de propagación de ondas, en particular, en el ancho de banda alrededor del modo fundamental y en las amplitudes y posiciones de los modos superiores de vibración del terreno.

En zonas densamente instrumentadas, las FTE de estaciones con valores grandes de $c_{p}(\sim 0.5)$ no deben influir significativamente en las interpolaciones. En zonas de insuficiencia instrumental, se busca 
que las interpolaciones dependan principalmente de los datos aislados ahí contenidos y de la información a priori (FTP). Al repetir este proceso para diferentes valores de $k$ entre 1 y 201 , se obtiene una FTP para el sitio de interés, cuyas abscisas son, como se ha señalado, periodos normalizados con respecto a Ts; la FTP cuyas abscisas son periodos absolutos de la excitación se obtiene reescalando las abscisas normalizadas.
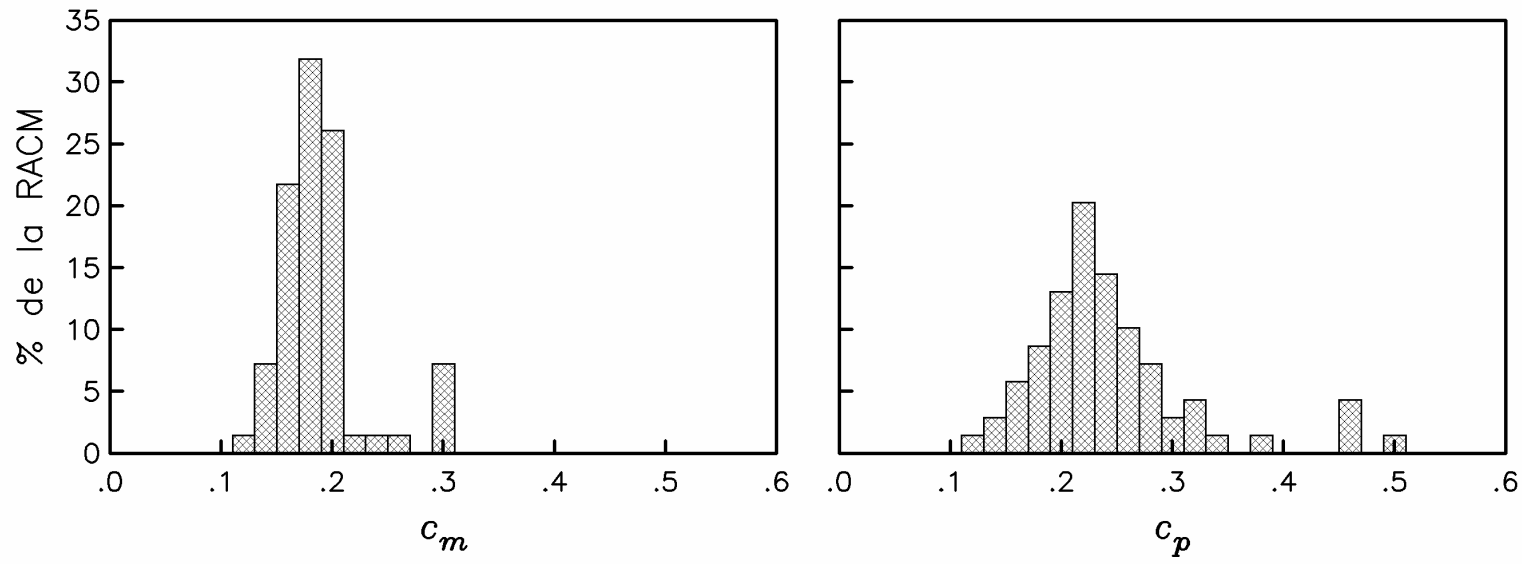

Figura 8 Histogramas de los coeficientes de variación $c_{m}$ (diferencias entre las FTE de varios temblores) $c_{p}$ (diferencia entre la FTP y la FTE promedio de varios temblores)
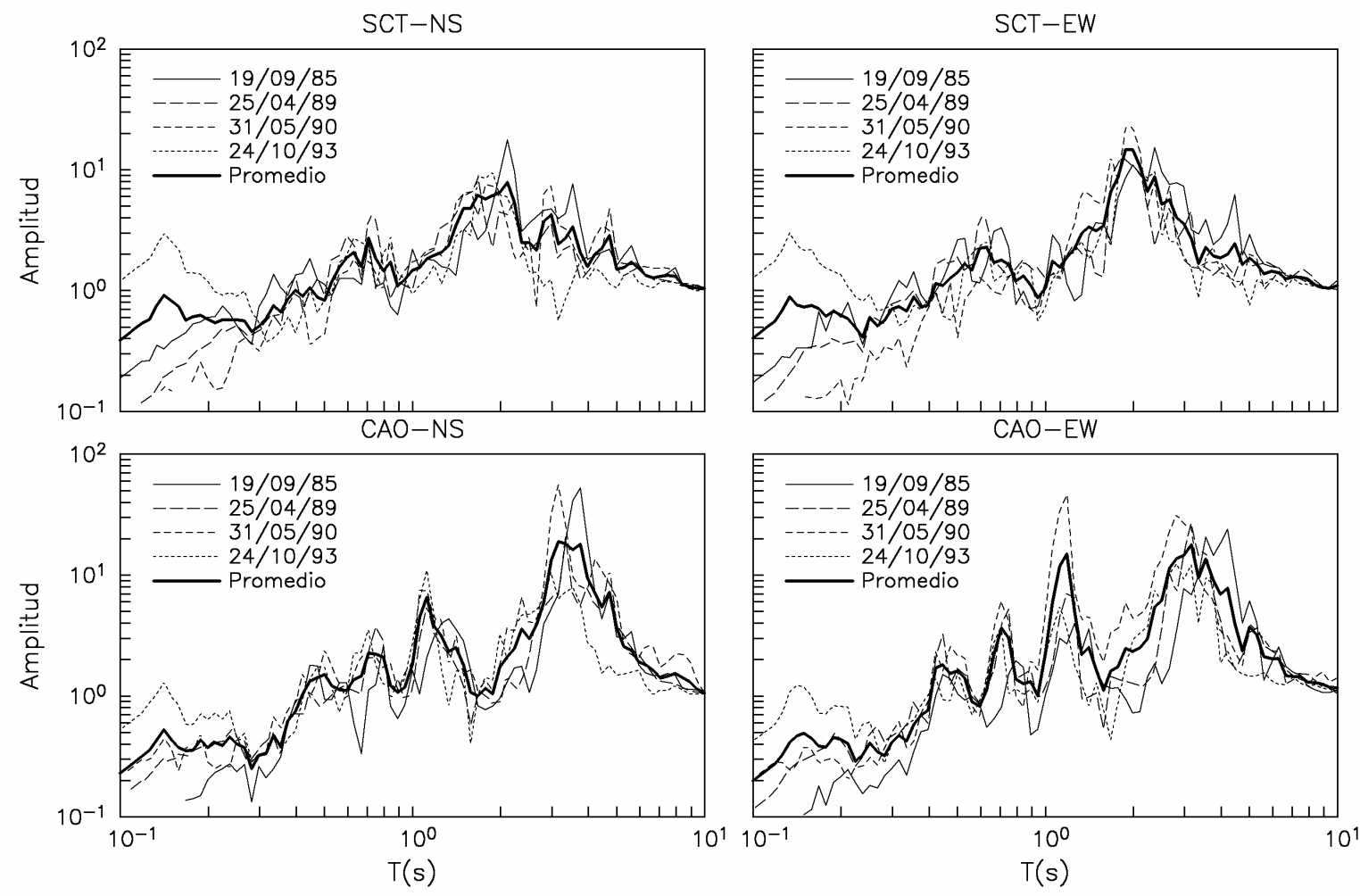

Figura 9 FTE para los sitios SCT (parte superior) y CAO (parte inferior), correspondientes a cuatro sismos, indicados con cuatro tipos de línea delgada. Con línea gruesa se indica el espectro promedio 

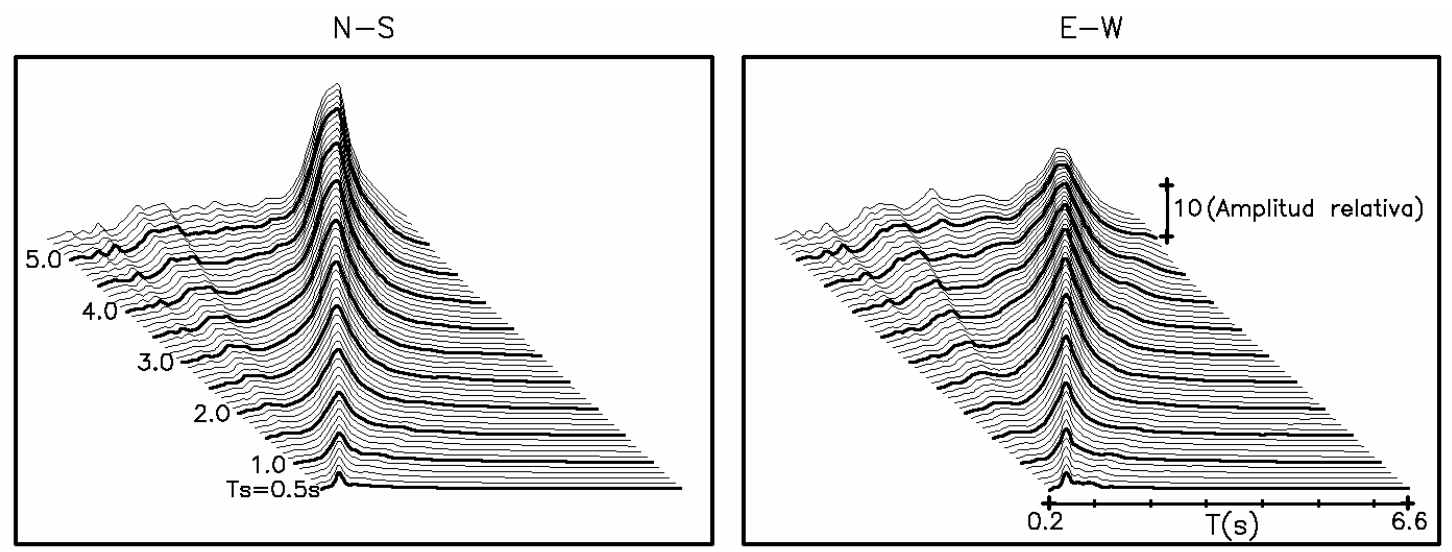

Figura 10 Funciones de transferencia a priori (FTP)

\section{Interpolación espacial de las FTE}

Para la interpolación espacial de las FTE se hace $c_{S i}=c_{p_{i}}$ en la ec (19). En este esquema también se introducen puntos de control que definen la frontera del terreno firme. Para estos sitios se propone $c_{S i}=0.125$. Una vez seleccionado el sitio de interés se calcula el periodo dominante del terreno Ts. Con este valor se determina la información a priori o FTP (componentes NS y EW) para cada valor de $\tau=\mathrm{T} / \mathrm{Ts}$.

Para la calibración de los parámetros $c_{\mathrm{r}}$ de la ec (19) y $s_{0}$, introducido para definir la matriz $\operatorname{COV}(\alpha)$, se calcularon las FTE considerando y excluyendo los datos en cada sitio instrumentado (modalidades con dato y sin dato, respectivamente). Para ambos casos se calcularon los coeficientes de variación esperados, siguiendo la ec (15), y los que se deben a las diferencias entre los datos y los valores interpolados en cada sitio instrumentado (llamados aquí errores o coeficientes de variación medidos). Estos coeficientes (denotados por $c_{a}, c_{b}, c_{c}, c_{d}$ ) corresponden a los que se definieron para la calibración del modelo de interpolación de los periodos dominantes del terreno. A la izquierda de la figura 11 se ilustra la variación de estos coeficientes con respecto a $c_{\mathrm{r}}$ haciendo $s_{0}=0.3$. A la derecha se ilustra la variación con respecto a $s_{0}$, haciendo $c_{\mathrm{r}}=0.1$. Se encontró que con los valores $c_{\mathrm{r}}=0.1 \mathrm{y} s_{0}=0.3$, los coeficientes de variación esperados con dato $\left(c_{b}\right)$ son comparables a los coeficientes $c_{m}$ en zonas densamente instrumentadas $\mathrm{y}$, además, que las FTE interpoladas en zonas insuficientemente instrumentadas son razonables y tienden adecuadamente a la solucione previa. En la figura, se observa que a medida que se incrementa el coeficiente $c_{\mathrm{r}}$, los errores medidos con dato y sin dato (indicados por los coeficientes $c_{a}$ y $c_{c}$, respectivamente) se reducen lentamente, mientras que los errores esperados para las modalidades con dato y sin dato (coeficientes $c_{b}$ y $c_{d}$, respectivamente) se incrementan rápidamente para valores de $c_{\mathrm{r}}>0.1$. Asimismo, al incrementar el coeficiente $s_{0}$, los errores medidos y esperados en la modalidad con dato (coeficientes $c_{a}$ y $c_{b}$, respectivamente) se reducen, mientras que los errores en la modalidad sin dato (coeficientes $c_{C} \mathrm{y} c_{d}$, respectivamente) se incrementan para valores de $s_{0}>0.2 \mathrm{y}$ $s_{0}>0.3$, respectivamente. 


\section{Interpolación espacial de las FTE}

Para la interpolación espacial de las FTE se hace $c_{S i}=c_{p_{i}}$ en la ec (19). En este esquema también se introducen puntos de control que definen la frontera del terreno firme. Para estos sitios se propone $c_{S i}=0.125$. Una vez seleccionado el sitio de interés se calcula el periodo dominante del terreno Ts. Con este valor se determina la información a priori o FTP (componentes NS y EW) para cada valor de $\tau=\mathrm{T} / \mathrm{Ts}$.

Para la calibración de los parámetros $c_{\mathrm{r}}$ de la ec (19) y $s_{0}$, introducido para definir la matriz $\operatorname{COV}(\alpha)$, se calcularon las FTE considerando y excluyendo los datos en cada sitio instrumentado (modalidades con dato y sin dato, respectivamente). Para ambos casos se calcularon los coeficientes de variación esperados, siguiendo la ec (15), y los que se deben a las diferencias entre los datos y los valores interpolados en cada sitio instrumentado (llamados aquí errores o coeficientes de variación medidos). Estos coeficientes (denotados por $c_{a}, c_{b}, c_{c}, c_{d}$ ) corresponden a los que se definieron para la calibración del modelo de interpolación de los periodos dominantes del terreno. A la izquierda de la figura 11 se ilustra la variación de estos coeficientes con respecto a $c_{\mathrm{r}}$ haciendo $s_{0}=0.3$. A la derecha se ilustra la variación con respecto a $s_{0}$, haciendo $c_{\mathrm{r}}=0.1$. Se encontró que con los valores $c_{\mathrm{r}}=0.1$ y $s_{0}=0.3$, los coeficientes de variación esperados con dato $\left(c_{b}\right)$ son comparables a los coeficientes $c_{m}$ en zonas densamente instrumentadas $\mathrm{y}$, además, que las FTE interpoladas en zonas insuficientemente instrumentadas son razonables y tienden adecuadamente a la solucione previa. En la figura, se observa que a medida que se incrementa el coeficiente $c_{\mathrm{r}}$, los errores medidos con dato y sin dato (indicados por los coeficientes $c_{a} \mathrm{y} c_{C}$, respectivamente) se reducen lentamente, mientras que los errores esperados para las modalidades con dato y sin dato (coeficientes $c_{b}$ y $c_{d}$, respectivamente) se incrementan rápidamente para valores de $c_{\mathrm{r}}>0.1$. Asimismo, al incrementar el coeficiente $s_{0}$, los errores medidos y esperados en la modalidad con dato (coeficientes $c_{a} \mathrm{y} c_{b}$, respectivamente) se reducen, mientras que los errores en la modalidad sin dato (coeficientes $c_{C}$ y $c_{d}$, respectivamente) se incrementan para valores de $s_{0}>0.2 \mathrm{y}$ $s_{0}>0.3$, respectivamente.

En la figura 12 se ilustran los histogramas de los errores medidos en las modalidades con dato y sin dato (coeficientes $c_{a}$ y $c_{c}$, respectivamente). De acuerdo con los resultados ilustrados a la izquierda, los coeficientes $c_{a}$ tienen media 0.1 y son menores que los coeficientes $c_{m}$ de la figura 8 . A la derecha se ilustra la distribución de los coeficientes $C_{C}$. Estos oscilan entre 0.08 y 0.5 , con media 0.2. Los histogramas de los errores esperados (coeficientes $c_{b}$ y $c_{d}$ ) se ilustran en la figura 13. En la modalidad con dato, los errores varían entre 0.1 y 0.45 , con media 0.23 . Por su parte, los errores sin dato varían entre 0.1 y 1.0 con media 0.32. Nótese que los errores esperados $\sin$ dato mayores que 0.5 se reducen substancialmente cuando se calculan en la modalidad con dato. Esto significa que, los errores esperados se sobrestiman sistemáticamente en zonas insuficientemente instrumentadas. 

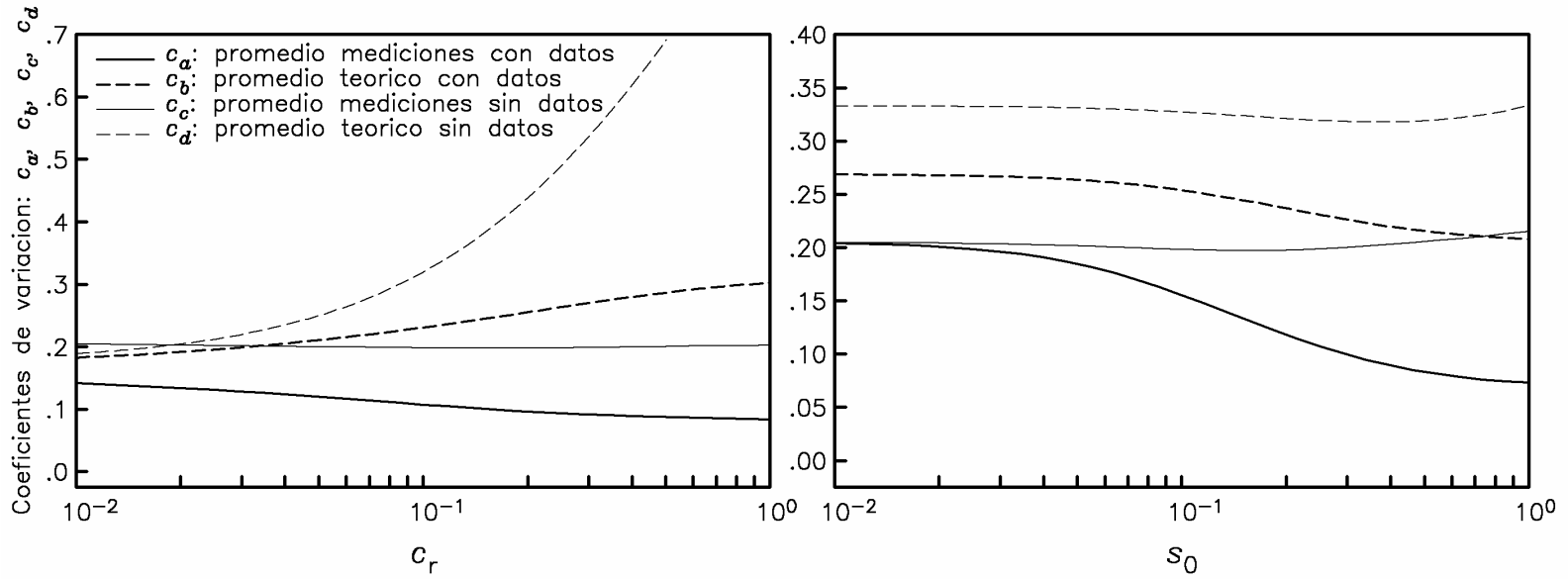

Figura 11 Coeficientes de variación relativos al error en la interpolación de las FTE
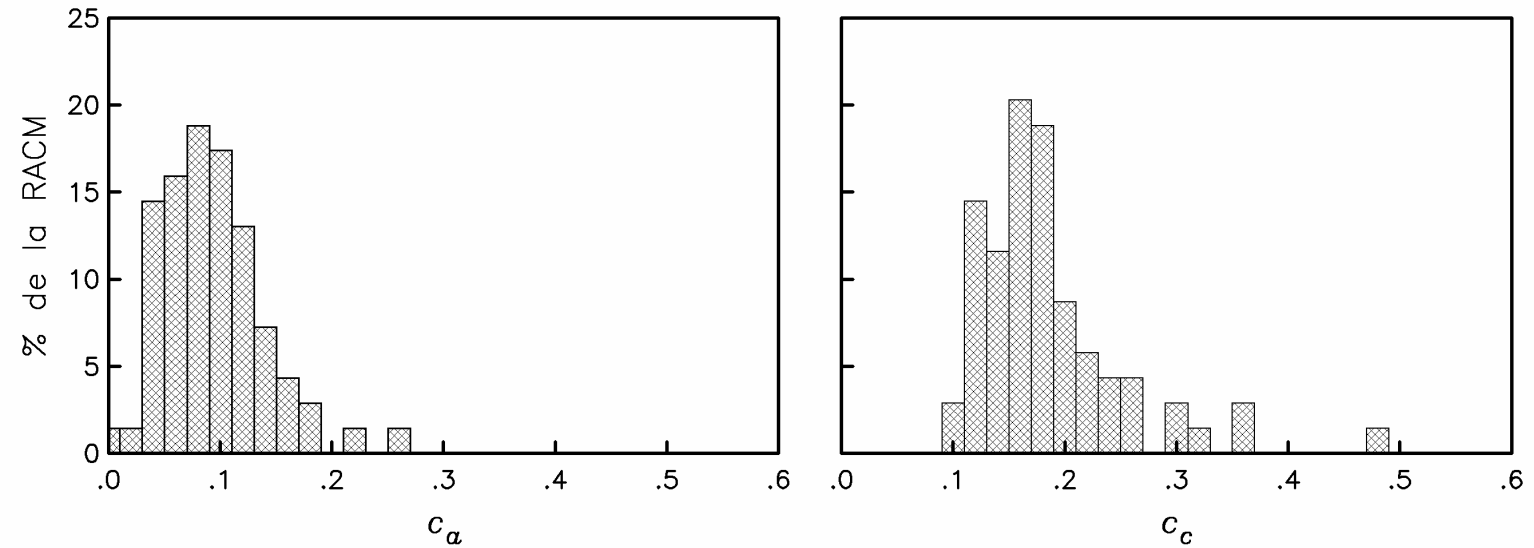

Figura 12 Coeficientes de variación medidos en las modalidades con dato $\left(c_{a}\right)$ y $\sin$ dato $\left(c_{c}\right)$
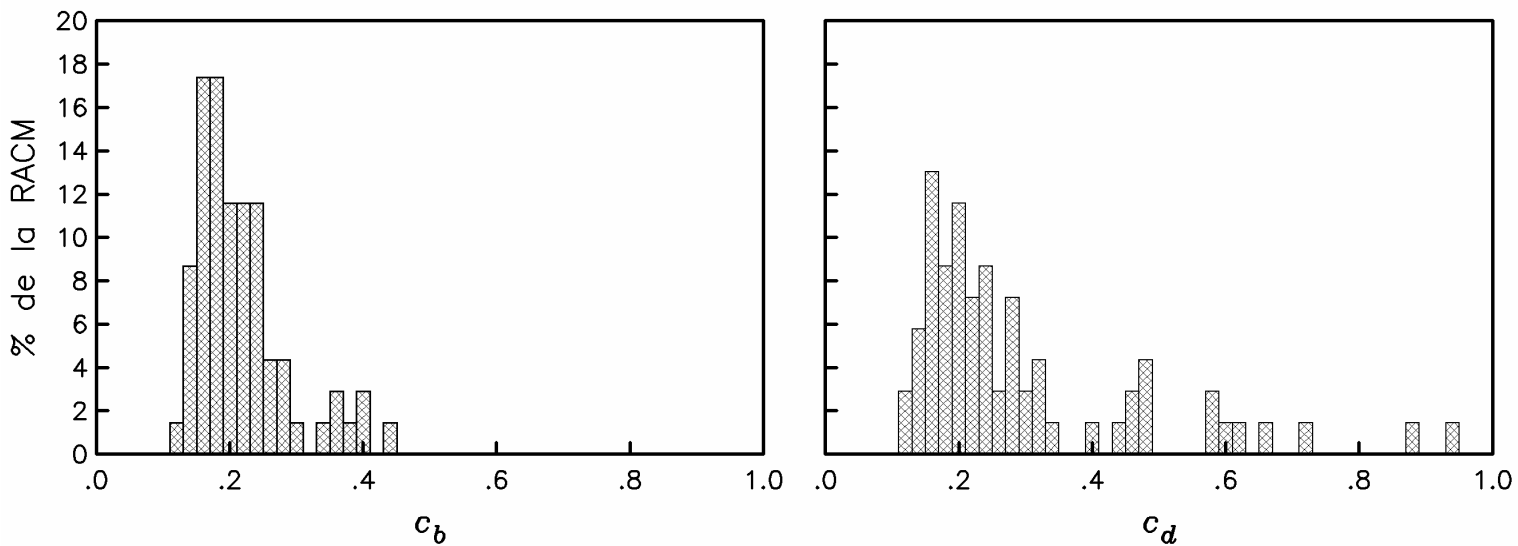

Figura 13 Coeficientes de variación esperados en las modalidades con dato $\left(c_{b}\right)$ y $\sin$ dato $\left(c_{d}\right)$ 
En la figura 14 se comparan las FTE observadas (líneas continuas) con las FTE interpoladas con dato (líneas discontinuas) y sin dato (líneas punteadas) para seis sitios en los que se obtuvieron errores con dato, medidos y esperados, menores que $10 \%$ y $20 \%$, respectivamente (ver figuras 12 y 13 ). Los resultados son excelentes. Por ejemplo, las estaciones 04 y 62 están en la zona con mayor densidad instrumental, separadas por $4 \mathrm{~km}$, aproximadamente. En estas estaciones los periodos del terreno son muy parecidos $(\mathrm{Ts}=1.95 \mathrm{~s}$ y $\mathrm{Ts}=2.05 \mathrm{~s}$, respectivamente). También lo son las funciones de transferencia, aunque se pueden identificar ligeras diferencias (principalmente el ancho de banda, las amplitudes máximas alrededor del periodo dominante y la forma del primer modo superior situado, aproximadamente, en $\mathrm{T}=0.8 \mathrm{~s}$ ). Al parecer, las interpolaciones sin dato reflejan extraordinariamente estas peculiaridades. Ello se debe a que la información a priori no es significativa en la interpolación de las FTE de estas estaciones.

En la figura 15 se hace la misma comparación para algunos de los sitios con los errores sin dato, medidos y esperados, mayores que 20\%. Para elegir estos sitios se buscó ilustrar la gama de periodos dominantes posibles para el terreno del valle de México. En todos los casos, las soluciones con dato y $\sin$ dato sugieren una combinación gradual entre las FTP y las FTE observadas. En la figura 16 se ilustran FTE correspondientes a los sitios con errores esperados sin dato mayores que $60 \%$ (estaciones 19,33 , tx y 14). Estos valores son un índice de los errores que se deben esperarse para las zonas menos instrumentadas. Los errores pueden ser mayores, pero no es desalentador al tomar en cuenta que para las estaciones más alejadas de la red $(19,33 \mathrm{y}$ tx) se tienen errores medidos con dato y sin dato bastante modestos. Los sitios referidos en las figuras 14,15 y 16 se localizan en la figura 1.

También se ilustran los beneficios que se tienen en la interpolación espacial al hacer uso de este modelo bayesiano con respecto a lo que se obtiene con el esquema convencional de mínimos cuadrados, tal como se expresa en las ecs 1 a 8. En la figura 17 se comparan las FTE observadas (líneas continuas) con las FTE interpoladas sin dato con dos variantes: siguiendo el modelo bayesiano (líneas discontinuas) y siguiendo el esquema convencional (líneas punteadas), para cuatro sitios localizados en la zona de suficiencia instrumental (estaciones $04,22,43$ y 62), con periodos que varían entre 1.5 y $3.0 \mathrm{~s}$. Se puede observar que, en estos sitios, ambos métodos proporcionan prácticamente los mismos resultados. En estas zonas de suficiencia instrumental, como se había anticipado, la información a priori no es significativa en la interpolación de las FTE.

En la figura 18 se hace la misma comparación, esta vez para sitios localizados en zonas de insuficiencia instrumental. Los sitios seleccionados son las estaciones 14, 19, 33 y Tx. Para las estaciones 14 y 19 aún se mantiene la estabilidad numérica en el esquema de interpolación convencional. Sin embargo, para las estaciones 33 y Tx se presentan inestabilidades numéricas que conducen a resultados en los que se comete el error mínimo pero que son físicamente incorrectos.

En la figura 19 se muestra un mapa con la distribución de los errores esperados en la interpolación espacial de las FTE ( $c_{V}=c_{C}$, sin excluir datos). Se han sombreado las áreas donde se esperan errores en los intervalos $0.2 \leq c_{V}<0.3,0.3 \leq c_{V}<0.5,0.5 \leq c_{V}<0.8$ y $c_{V} \geq 0.8$. Para la zona de terreno firme, el error esperado es $c_{V}=0.125$. Nótese que en la zona del lago, los errores menores se esperan en el área con el mayor índice instrumental, mientras que en las regiones no instrumentadas los errores son $c_{V} \geq 0.8$. Estos valores pueden llegar a $c_{v} \geq 1.25$. 

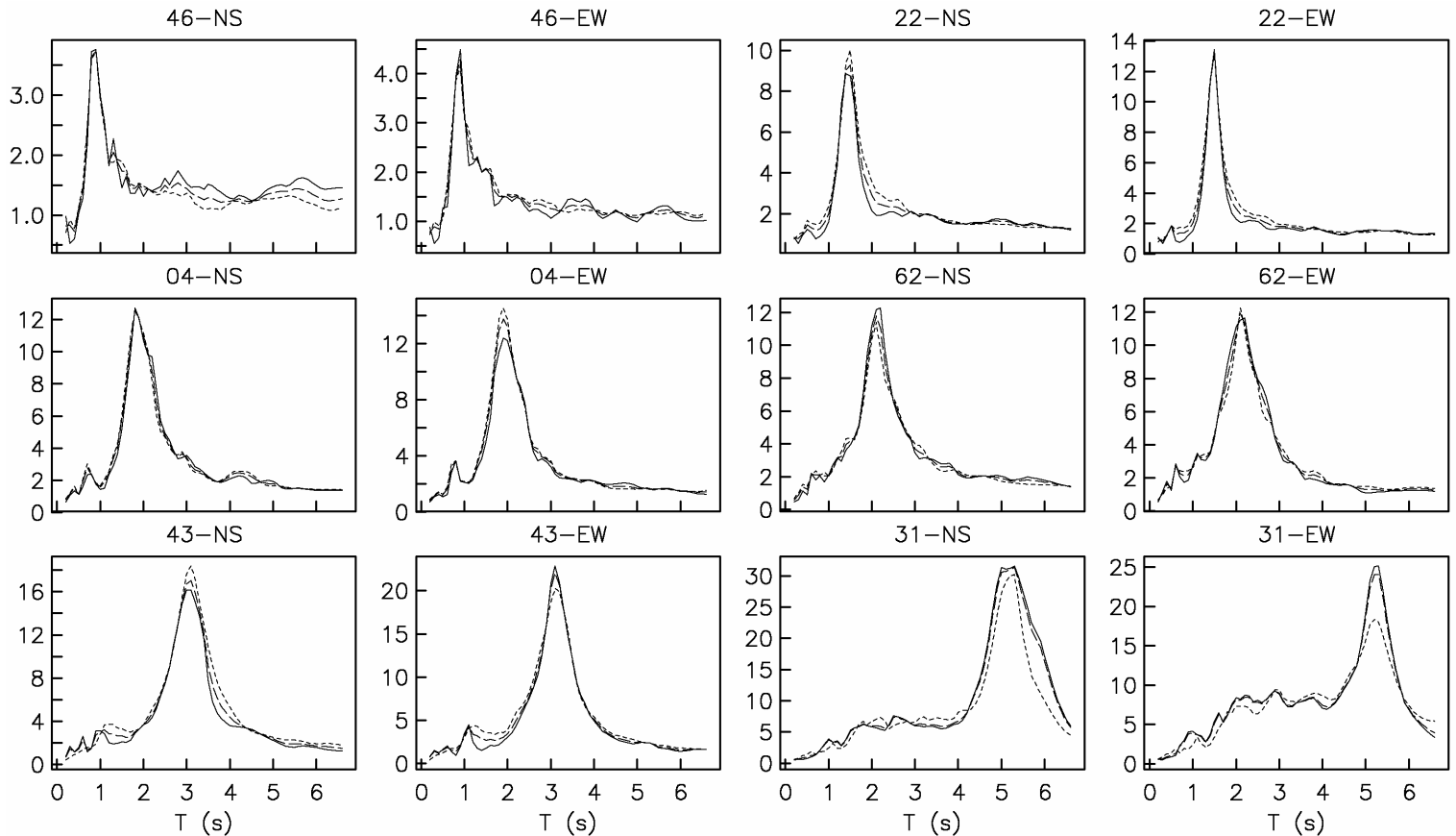

Figura 14 FTE observadas (líneas continuas) y FTE interpoladas con dato (líneas discontinuas) y sin dato (líneas punteadas) para sitios con errores con dato, medidos y esperados, menores que $10 \% \mathrm{y}$ $20 \%$.
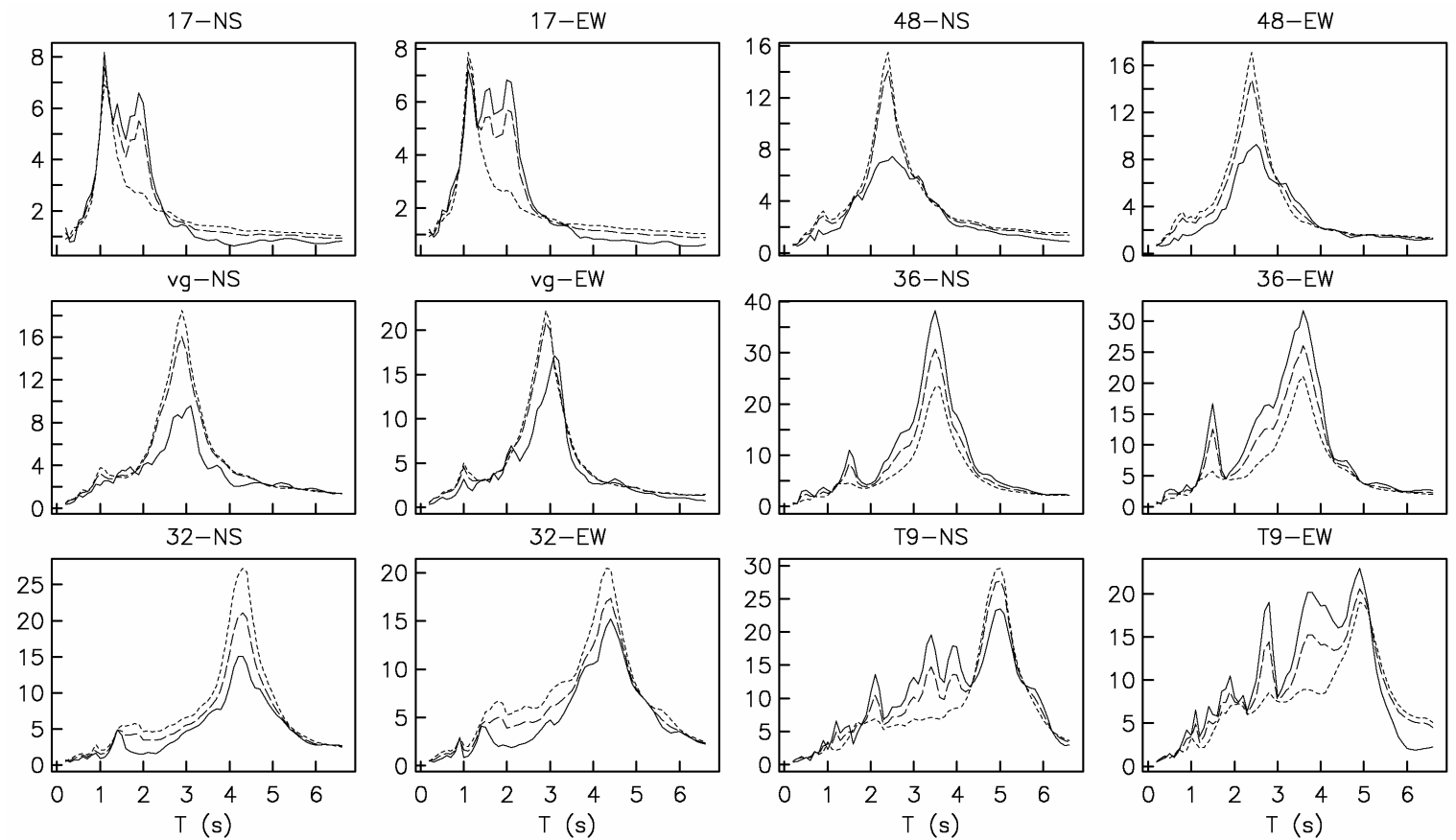

Figura 15 Igual que figura 14 pero para sitios con errores con dato, medidos y esperados, mayores que 10 $\%$ y $20 \%$, respectivamente. 

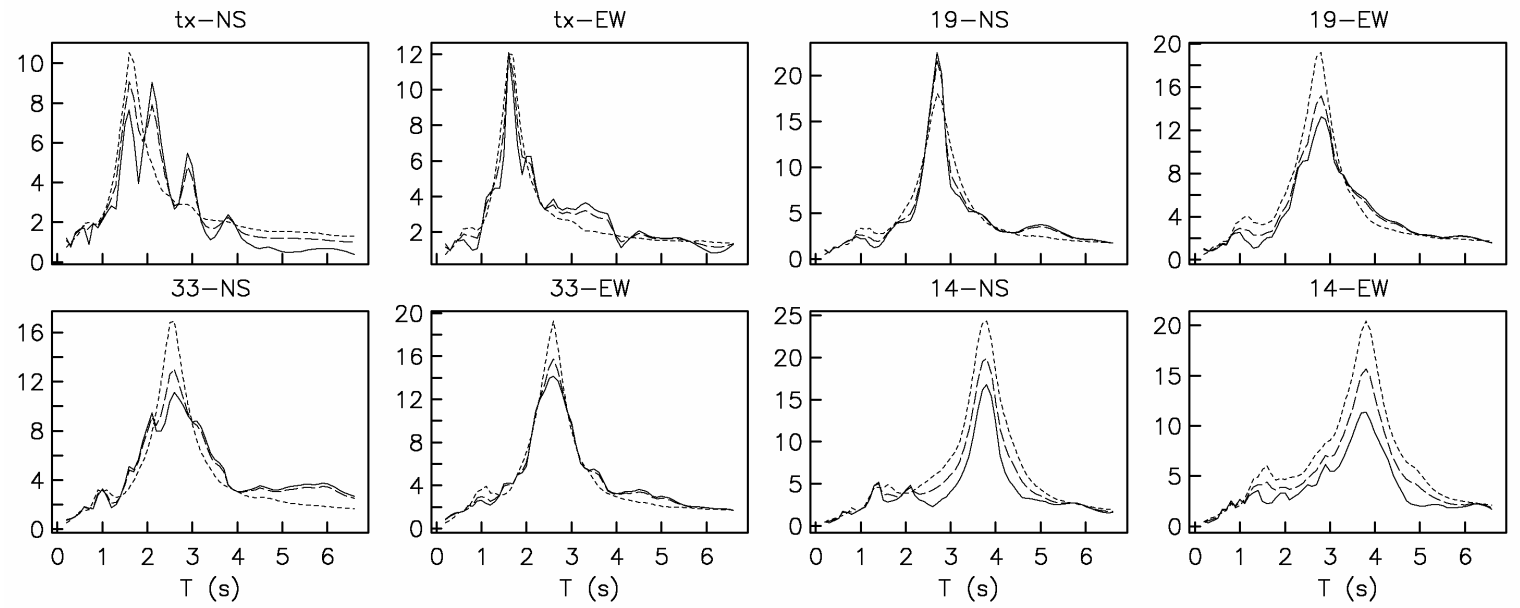

Figura 16 Igual que figura 14, para sitios con errores esperados sin dato mayores que $60 \%$ (estaciones aisladas)
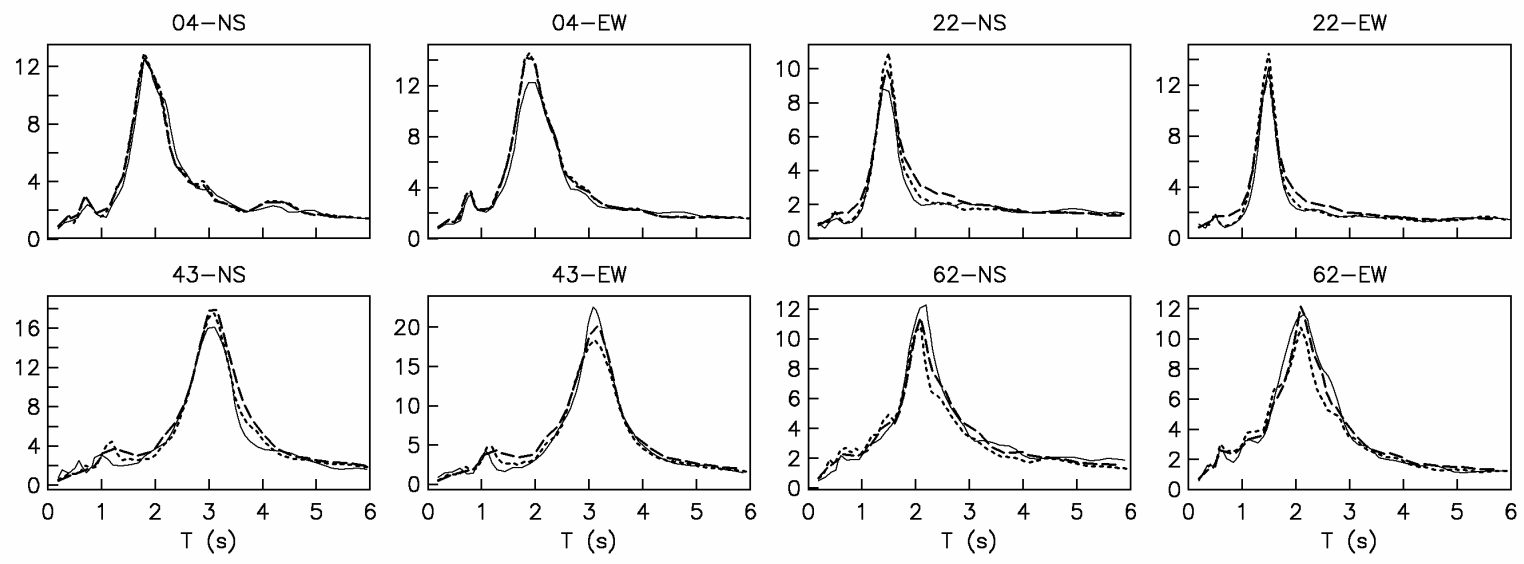

Figura 17 FTE observadas (líneas continuas) y FTE interpoladas sin dato siguiendo dos esquemas: bayesiano (discontinuas) y convencional (punteadas) para sitios localizados en la zona de suficiencia instrumental
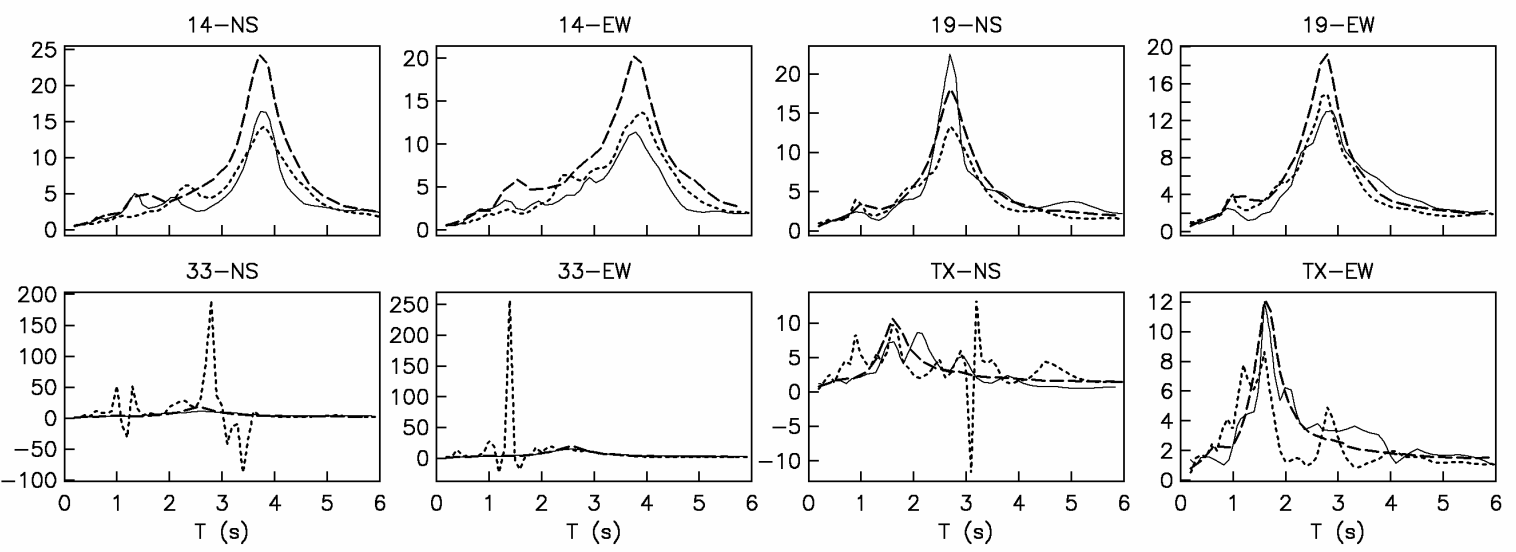

Figura 18 Igual que figura 17 pero para sitios localizados en la zona de insuficiencia instrumental 


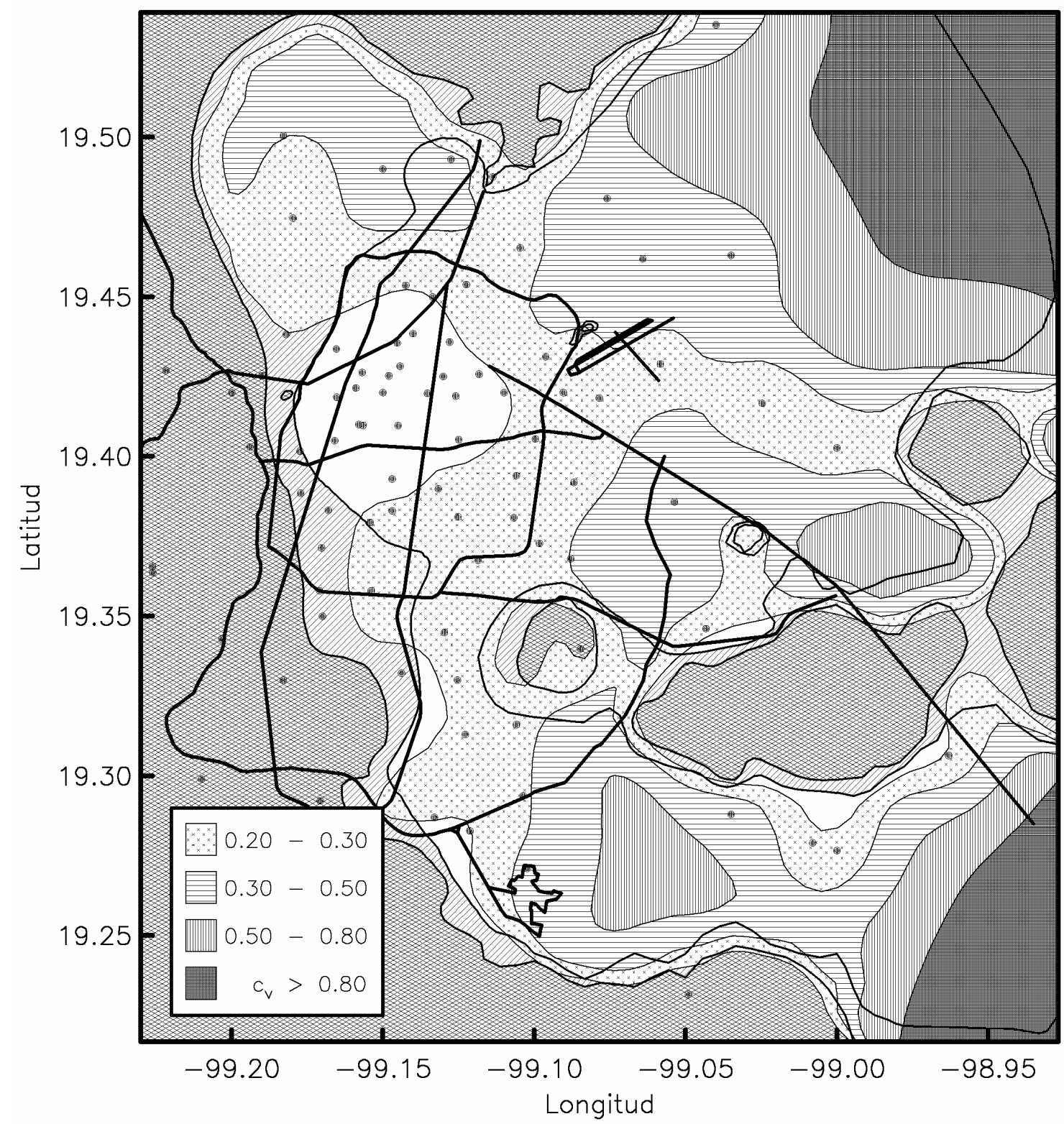

Figura 19 Coeficientes de variación esperados en la interpolación defunciones de transferencia empíricas

En la figura 20 se muestran las FTE interpoladas a lo largo de una sección entre las estaciones 52 y 31. Estas estaciones tienen periodos dominantes de $T s=0.8 \mathrm{~s}$ y $T \mathrm{~s}=5.3 \mathrm{~s}$, respectivamente. La sección cruza el área con el menor índice de error. Entre otros aspectos, puede verificarse que las variaciones espaciales son suaves y que existen diferencias significativas entre los componentes NS y EW. En la figura 21 se presentan las FTE interpoladas a lo largo de una sección entre la estaciones T9 y un sitio arbitrario (SA) con coordenadas $-98.95 \mathrm{~W}, 19.50 \mathrm{~N}$. Los periodos dominantes son $\mathrm{Ts}=5.1 \mathrm{~s}$ y $\mathrm{Ts}=1.2 \mathrm{~s}$, respectivamente. Esta sección se tiende sobre el área con el mayor índice de error. Se puede verificar que la solución está controlada por las FTP en mayor cuantía a medida que aumenta la distancia a la estación T9 (ver figura 9). Finalmente, en la figura 22 se ilustran las FTE interpoladas a lo largo de una sección entre las estaciones 15 y 35. Esta sección se escogió porque cruza el área donde se presentan las mayores inestabilidades numéricas cuando el coeficiente de variación relativo a la información a priori es inadecuadamente grande 
$\left(s_{0} \sim 1.0\right)$. Si el coeficiente es menor $\left(s_{0} \sim 0.3\right)$ se obtienen resultados razonables y congruentes con naturaleza física y con las observaciones.
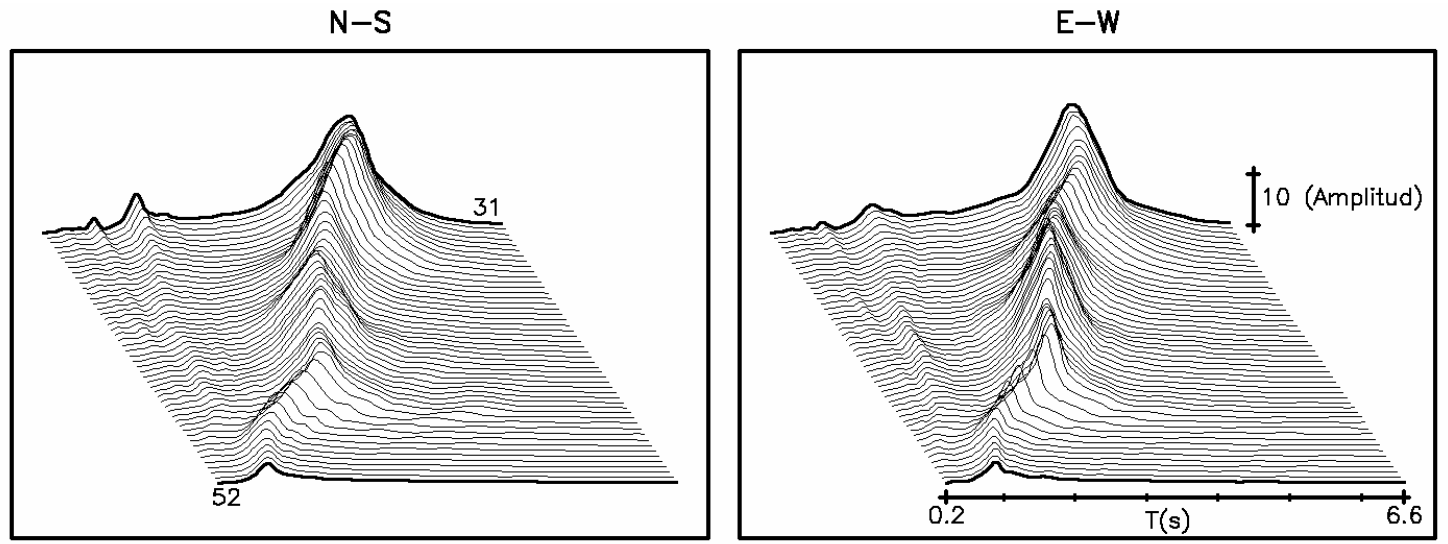

Figura 20 FTE interpoladas a lo largo de una sección entre las estaciones 52 y 31
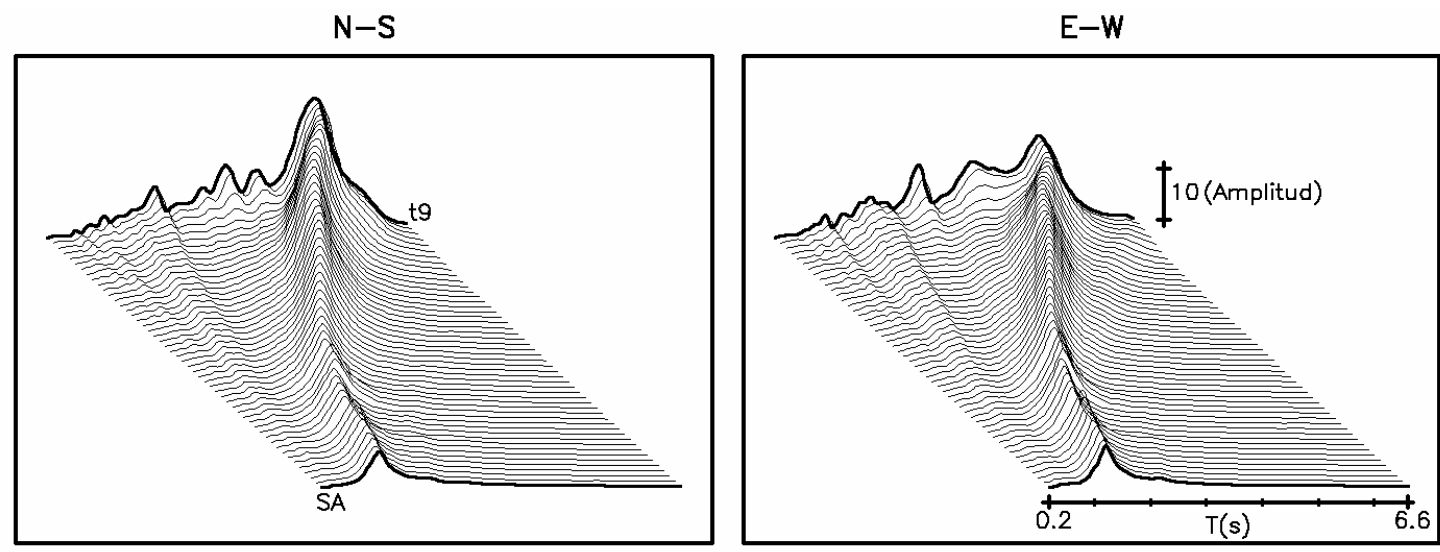

Figura 21 FTE interpoladas a lo largo de una sección entre el sitio SA y la estación t9
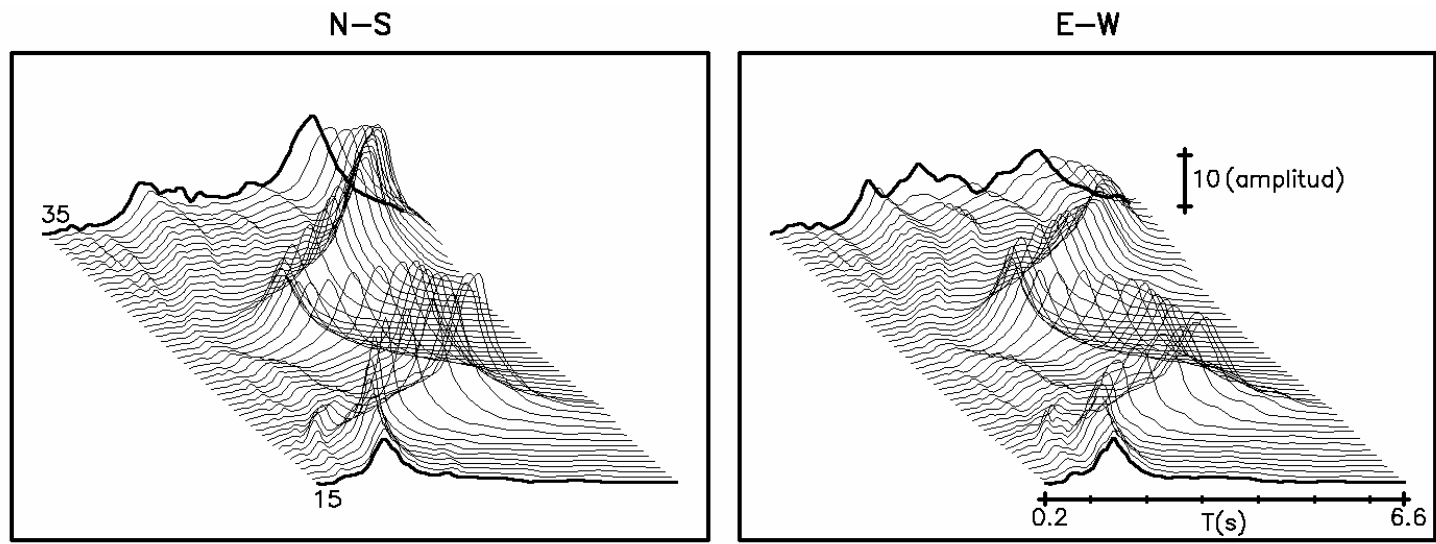

Figura 22 FTE interpoladas a lo largo de una sección entre las estaciones 15 y 35 


\section{CONCLUSIONES}

Se ha descrito un método para resolver un problema de interpolación espacial. Los datos son las funciones de transferencia empíricas que se han obtenido de los acelerogramas de varios temblores registrados en decenas de sitios en las zonas de terreno firme, transición y lago del valle de México. El planteamiento de un esquema estadístico para tratar la interpolación espacial de las funciones de transferencia mediante el uso de las técnicas de regresión bayesiana, ha permitido incorporar formalmente la información que se tiene acerca de los efectos de sitio en la respuesta dinámica del terreno. Esta formulación suministra soluciones más estables y racionales que las que se obtendrían al seguir esquemas de regresión convencionales para la interpolación espacial de datos geofísicos.

Para cada estación, se interpolaron las FTE (dos componentes horizontales) y se compararon con las FTE medidas en los registros (llamadas FTE observadas). Se encontró que las diferencias entre estas FTE son menores que las que se observan en las FTE producidas por un temblor y por otro. También se identificaron los errores que se cometen por el hecho de interpolar las FTE en sitios no instrumentados. Para ello, se calcularon las FTE de cada estación sin considerar los temblores registrados en ella y se compararon con las FTE observadas. Estas modalidades en el cálculo de FTE se identifican como con dato y sin dato, respectivamente. Se encontró que en las zonas densamente instrumentadas, los errores esperados en la interpolación de las FTE son del orden del $20 \%$, en tanto que en las zonas insuficientemente instrumentadas pueden ser del 100\%.

Como resultado parcial, se obtuvo un mapa de periodos dominantes del terreno, con base en toda la información proveniente de registros de movimiento fuerte y de microtremores, así como una medida de los errores cometidos en la interpolación de estas cantidades. Para la zona con mayor densidad instrumental, los errores pueden ser menores que $5 \%$ y pueden llegar al $70 \%$ en las zonas con instrumentación deficiente.

\section{REFERENCIAS}

Broemling L D (1985). Bayesian Analysis of Linear Models. Marcel Dekker, Inc., New York.

Bufaliza M (1984). "Atenuación de intensidades sísmicas con la distancia en sismos mexicanos”, Tesis de maestría, Facultad de Ingeniería UNAM.

Castro R, S K Singh y E Mena (1988). "An empirical model to predict Fourier amplitude spectra of horizontal ground motion", Earthquake Spectra, 4, 675-686.

Esteva L y R Villaverde (1974). "Seismic risk, design spectra and structural reliability", Memorias del V Congreso Mundial de Ingeniería Sísmica, Roma, Italia, 2586-2597.

Lancaster P y K Salkauskas (1986). Curve and surface fitting. An introduction. Academy Press, Londres.

Lermo J, M Rodríguez y S K Singh (1988). "Natural period of sites in the Valley of Mexico from microtremor measurements". Earthquake Spectra, 4, 805-814.

Ordaz M, E Reinoso, S K Singh, E Vera y J M Jara (1989). "Espectros de respuesta en diversos sitios del Valle ante temblores postulados en la brecha de Guerrero", Memorias del VIII Congreso Nacional de Ingeniería Sísmica y VII Congreso Nacional de Ingeniería Estructural, Acapulco, México, A187-A198. 
Ordaz M y S K Singh (1992). "Source spectra and spectral attenuation of seismic waves from Mexican earthquakes and evidence of amplification in the hill zone of Mexico City", Bull Seism Soc Am, 82, 24-43.

Ordaz M, R Meli, C Montoya-Dulché, L Sánchez y L E Pérez-Rocha (1992). "Data base for seismic risk assessment in México City". Simposio Internacional sobre Prevención de Desastres Sísmicos. Centro Nacional de Prevención de Desastres, Mayo 18-21. México.

Ordaz M, Singh S K y Arciniega A (1994). "Bayesian Attenuation regressions: an application to México City", Geophys J Int, 117, 335-344.

Pelto C R, Elkins T A y Boyd H A (1988). "Automatic contouring of irregularly spaced data", Geophysics, 33, 424-430.

Pérez-Rocha L E, R Aguilar, A Arciniega, E Reinoso, M Ordaz y F J Sánchez-Sesma (1991). "Respuesta sísmica del valle de México: Aplicaciones y Teoría". Informe Final presentado a la Secretaría de Obras del Despartamento del Distrito Federal.

Pérez-Rocha L E, M Ordaz y F J Sánchez-Sesma (1995). “Interpolación espacial de datos sísmicos”. Congreso Panamericano de Cimentaciones. Guadalajara, Jal. México.

Pérez-Rocha L E, L Viéitez, F Flores y M Zárate (1996). "Predicción de intensidades sísmicas para el área metropolitana del Valle de México”. Cuadernos FICA \# 13.

Reinoso E (1991). "Efectos sísmicos locales en el Valle de México: amplificación medida en la zona lacustre”. Mem 9a. Conf Nal de Ing Sísm, 2, 224-236, Manzanillo, México.

Reinoso E, L E Pérez-Rocha, M Ordaz y A Arciniega (1992). "Prediction of response spectra at any site in Mexico City”. Mem 10a. Conf Int de Ing Sísm, 767-772, Madrid, España.

Sánchez-Sesma F J, L E Pérez-Rocha y E Reinoso (1992). "Ground motion in México City during the April 25, 1989 Guerrero earthquake". Tectonophysics, 218, 127-140.

Singh S K, Mena E, Castro R y Carmona C (1987). "Empirical Prediction of ground motion in Mexico City from coastal earthquakes”, Bull Seism Soc Am, 78, 230-242.

Singh S K, Lermo J, Domínguez T, Ordaz M, Espinosa J M, Mena E y Quaas R (1988). "A study of amplification of seismic waves in the Valley of Mexico with respect to a hill zone site". Earthquake Spectra, 4, 653-674. 